\title{
Integral de Kurzweil-Henstock em um disco p-ádico
}

José Angelo Pezzotta

\author{
Tese apresentada \\ ao \\ Instituto de Matemática e Estatística \\ da \\ Universidade de São Paulo \\ para \\ obtenção do grau \\ de \\ Doutor em Matemática
}

Área de Concentração: ANÁLISE

Orientador:Prof. Dr. RICARDO BIANCONI

São Paulo-agosto-2005 


\title{
Integral de Kurzweil-Henstock \\ em um disco p-ádico
}

\author{
Este exemplar corresponde à redação final da tese \\ devidamente corrigida e defendida por \\ José Angelo Pezzotta \\ e aprovada pela comissão julgadora.
}

São Paulo, dezembro de 2005

Comissão Julgadora:

- Prof. Dr. Ricardo Bianconi (Presidente)- IME-USP

- Prof. Dr. Paulo Agozzini Martin - IME-USP

- Prof. Dr. Plácido Zoega Taboas - ICMC-USP

- Prof. Dr. José Luiz Boldrini - LNICAMP

- Prof. Dr. Dicesar Lass Fernandes - UNICA.MP 
À minha cara amiga e mestre

Profte Dra Iracema Martin Bund

com carinho e respeito. 


\section{Agradecimentos}

Ao Prof. Dr. Ricardo Bianconi os nossos sinceros agradecimentos por ter aceitado orientar-nos neste trabalho. Sua dedicada orientação, amizade, apoio, sempre apresentando sugestões e corrigindo os nossos erros (alguns crassos) sempre com bom humor e paciência tornaram o nosso caminho nesta empreitada mais suave.

À minha querida esposa, Paula, que durante todo o tempo que dedicamos ao desenvolvimento desta pesquisa, sempre procurou dar a sua contribuição, através do incentivo e compreensão, a minha gratidão e o meu carinho.

Ao Prof. Dr. Chaim Samuel Hönig, com o qual tomei o primeiro contato com a integral de Kurzweil-Henstock, a minha gratidão. 


\title{
Resumo
}

Neste trabalho desenvolvemos a integral de Kurzweil-Henstock em um disco p-ádico. Apresentamos a sua definição; provamos suas propriedades elementares; verificamos o Critério de Cauchy e o Lema de Henstock; estudamos os principais teoremas de convergência: o Teorema da Convergência Monótona e o Teorema da Convergência Dominada de Lebesgue. Introduzimos os conceitos de funções e conjuntos mensuráveis, definimos uma medida no disco p-ádico e provamos que a mesma é uma medida de Haar. Estabeleccmos relações entre funções mensuráveis e funções integráveis e finalizamos resolvendo a equação de Fredholm de $2^{\underline{a}}$ espécie no disco p-ádico.

\begin{abstract}
In this work we develop the Kurzweil-Henstock integral in a p-adic disk. We introduce its definition and we prove its basic properties, the Cauchy criterion and the Henstock's Lemma. We study the principal theorems of convergence: the Monotone Convergence Theorem and the Dominated Convergence Theorem. The concepts of measurable functions and measurable sets are introduced, we define one measure in the p-adic disk and we prove that it is the Haar measure. We establish relations between measure functions and integrable functions and we finish solveing the 2 th Fredholm equation in the p-adic disk.
\end{abstract}




\section{Sumário}

1 Preliminares 4

1.1 Espaços ultramétricos . . . . . . . . . . . . . . . 4

1.2 Números p-ádicos . . . . . . . . . . . . . . 6

2 Integral de Kurzweil-Henstock 13

2.1 Introdução . . . . . . . . . . . . . . . . . . . . . . . 13

2.2 A integral como funcional linear . . . . . . . . . . . . . . 19

2.3 O Critério de Cauchy . . . . . . . . . . . . . . . 20

2.4 A integral como função de discos . . . . . . . . . . 22

2.5 A integral e as funções contínuas . . . . . . . . . 25

2.6 O Lema de Henstock . . . . . . . . . . . . . . . . 28

2.7 As funções absolutamente integráveis . . . . . . . . . . 31

3 Teoremas de Convergência 36

3.1 Conjunto Nulo . . . . . . . . . . . . . . . . 36

3.2 Teoremas de Convergência . . . . . . . . . . . 38

4 Integração em conjuntos 51

4.1 Funções mensuráveis . . . . . . . . . . . . . . . 51

4.2 Conjuntos mensuráveis . . . . . . . . . . . . . . . 54

4.3 Integrabilidade e mensurabilidade . . . . . . . . . . 60

5 Aplicação da Integral $\quad 70$

5.1 Equação de Fredholm . . . . . . . . . . . . . . . 70 


\section{Prefácio}

Em 1957 Jaroslav Kurzweil apresentou uma definição de integral que se mostrou, em diversos aspectos, mais abrangente que aquela de Lebesgue. Posteriormente, Ralph Henstock desenvolveu a teoria dessa nova integral que passou a ser apresentada com os nomes de integral de Kurzweil, integral de Henstock, integral de Riemann generalizada ou integral de gauge. Em respeito e homenagem aos pioneiros desta nova ferramenta da matemática, chamamos neste trabalho, a integral de Kurweil-Henstock.

Nosso interesse por essa integral surgiu quando participamos de um seminário ministrado pelo Prof. Dr. Chaim S. Hönig no IME na década de 1990, e a oportunidade de pesquisarmos sobre a mesma surgiu através do Prof. Dr. Ricardo Bianconi que se prontificou em orientar-nos nessa pesquisa.

O objetivo deste trabalho é desenvolver a integral de Kurzweil-Henstock em um disco contido no conjunto dos números p-ádicos.

Certamente a curiosidade do leitor indagará sobre a motivação para desenvolver tal integral no disco p-ádico. Desde a época de Euclides o espaço euclidiano $\mathbb{R}^{3}$ é tomado como o modelo matemático para o espaço físico real. Êle é um modelo tão bom que facilmente verificamos na prática os seus axiomas e teoremas. Entretanto está provado que em gravidade quantum e teoria das molas há embraços para se realizar medidas tomando-se o espaço euclidiano como modelo matemático, uma vez que não se pode medir segmentos cujos comprimentos são inferiores ao denominado comprimento de Planck, que é da ordem de $10^{-33} \mathrm{~cm}$. Isso significa afirmar que o modelo matemático para espaços físicos cujas distâncias apresentam medidas muito pequenas não pode ser arquimediano ou, em outras palavras, os números reais não são adequados para as medidas de comprimento nesses espaços. Por outro lado, no nosso cotidiano, qualquer ação prática que exija uma medida e qualquer que seja o aparelho utilizado para expressá-la e, ainda mais, por mais preciso que seja esse aparelho, o resultado da medição será sempre um número racional. 
Assim, para resolvermos problemas da geometria das pequenas distâncias devemos considerar um conjunto que contenha todos os números racionais e, além disso, deve possuir a propriedade de não ser arquimediano. O conjunto dos números p-ádicos contém todos os números racionais e é não arquimediano, logo ele pode ser um modelo para essa geometria. Esse fato motivou-nos o desenvolvimento da integral de Kurzweil-Henstock no disco p-ádico. Como os conhecimentos sobre os números p-ádicos e sua estrutura topológica são necessários para a construção da integral apresentamos, no capítulo primeiro, os resultados essenciais sobre espaços ultramétricos e números p-ádicos. Todos os resultados apresentados são conhecidos, entretanto optamos por deixar a demonstração daqueles que foram mais utilizados no texto.

No capítulo segundo iniciamos a construção da integral de KurzweilHenstock propriamente dita. Nessa construção procuramos seguir o desenvolvimento lógico apresentados pelos livros sobre o tema no conjunto dos números reais. Aqui, [21] e [34] serviram de referência. Nesse capítulo apresentamos a definição e as propriedades básicas da integral, o critério de Cauchy e o Lema de Henstock. A integrabilidade das funções contínuas e as condições para que uma função seja absolutamente integrável também são vistas. Alguns exemplos ilustram este capítulo.

O capítulo 3 reservamos para os teoremas de convergência. Ele inicia-se com o conceito de conjunto nulo para que possamos enunciar os teoremas importantes de convergência: Teorema da Convergência Monótona e Teorema da Convergência Dominada utilizando a propriedade válida quase sempre.

No capítulo 4 a definição de funções mensuráveis é fornecida por Bartle em [1]. Nele mostramos que toda função contínua é mensurável; construímos a sigma-álgebra dos conjuntos mensuráveis, definimos a medida de um conjunto e mostramos que essa medida é de Haar. Mostramos a existência de conjuntos não mensuráveis e encerramos o capítulo mostrando que toda função integrável é mensurável.

No capítulo 5 efetuamos uma aplicação da integral resolvendo a equação de Fredholm de $2^{\underline{a}}$ espécie no disco p-ádico.

Finalmente, embora o conjunto dos números p-ádicos apresentem uma topologia estranha e não possa ser linearmente ordenado, observamos a existência de teoremas com demonstrações semelhantes a aquelas presentes no intervalo real.

As notações utilizadas são apresentadas no decorrer do texto. 


\section{Capítulo 1}

\section{Preliminares}

Neste capítulo apresentaremos os conceitos básicos necessários para o desenvolvimento dos nossos trabalhos. Esses resultados são conhecidos e podem ser encontrados em [14], [19] ou [36].

\subsection{Espaços ultramétricos}

Definição 1.1. Seja E um conjunto não vazio. L'ma ultramétrica em E é uma função real $d: E \times E \rightarrow] 0, \infty$ [ que associa a cada par ordenado $(x, y) \in E \times E$ o número real $d(x, y) \geq 0$, denominado distância de $x$ a $y$, satisfazendo as condições abaixo, quaisquer que sejam $x, y, z \in E$ :

(i) $\quad d(x, y)=0$ se e somente se $x=y$;

(ii) $\quad d(x, y)=d(y, x)$;

(iii) $d(x, y) \leq \max \{d(x, z), d(z, y)\}$.

O par (E,d) é chamado de espaço ultramétrico e cada elemento de E é um ponto desse espaço. Nesta seção reservaremos a letra E para designar um espaço ultramétrico com uma métrica já estabelecida. Somente faremos menção explícita ao espaço ultramétrico E ou à métrica quando necessário. 
Proposição 1.2. Se $x, y, z \in E$ são tais que $d(x, z) \neq d(z, y)$, então $d(x, y)=\max \{d(x, z), d(z, y)\}$.

Corolário 1.3. Quaisquer que sejam $x, y, z \in E$ tem-se: $d(x, y)=d(x, z)$ ou $d(x, y)=d(z, y)$ ou $d(x, z)=d(z, y)$. les.

Segue-se de (1.3) que, em um espaço ultramétrico, todo triângulo é isósce-

Notações 1.4. Dado o número real $r \geq 0$, o conjunto $\{z \in E: d(x, z) \leq r\}$ é chamado de disco de centro $x$ e raio $r$ e é denotado por $D_{r}(x)$. Se $r>0$, o conjunto $\{z \in E: d(x, z)<r\}$ é chamado de bola aberta de centro $x$ e raio $r$ e é denotado por $B_{r}(x)$.

Proposição 1.5. Quaisquer que sejam $x, y \in E$ e $r, s \in] 0, \infty[$, com $s \geq r$, tem-se:

(i) se $D_{r}(x) \cap D_{s}(y) \neq \emptyset$, então $D_{r}(x) \subset D_{s}(y)$;

(ii) se $B_{r}(x) \cap B_{s}(y) \neq \emptyset$, então $B_{r}(x) \subset B_{s}(y)$.

Corolário 1.6. Quaisquer que sejam $x, y \in E$ e $r \in] 0, \infty[$, tem-se:

(i) se $D_{r}(x) \cap D_{r}(y) \neq \emptyset$, então $D_{r}(x)=D_{r}(y)$;

(ii) se $B_{r}(x) \cap B_{r}(y) \neq \emptyset$, então $B_{r}(x)=B_{r}(y)$

De (1.6) segue-se que todo ponto pertencente a uma bola aberta ou a um disco é um centro da bola ou do disco, respectivamente. Em vista disso, quando não houver possibilidade de confusão, denotaremos uma bola aberta ou um disco de raio $r$, simplesmente, por $B_{r}$ ou $D_{r}$, respectivamente.

Decorre ainda dessa proposição que, para todo $r \in] 0, \infty[$, a coleção das bolas abertas ou dos discos de raio $r$ é uma partição de E. 
Corolário 1.7. Quaisquer que sejam $r_{1}, \ldots, r_{n}$ números reais positivos satisfazendo $r_{i} \leq r$, para todo $1 \leq i \leq n$, tem-se:

(i) se $D_{r} \subset \bigcup_{i=1}^{n} D_{r_{i}}$, então existe $m, 1 \leq m \leq n$, tal que $D_{r}=\bigcup_{\substack{i=1 \\ m}}^{m} D_{r_{i}}$;

(ii) se $B_{r} \subset \bigcup_{i=1}^{n} B_{r_{1}}$, então existe $m, 1 \leq m \leq n$, tal que $B_{r}=\bigcup_{i=1}^{m} B_{r_{i}}$.

Definição 1.8. Seja $A \subset E$. Diz-se que A é limitado se existe um númcro real $M>0$ tal que, quaisquer que sejam $x, y \in A$, tem-se $d(x, y) \leq M$. Se A é limitado, o número real $\rho(A)$, definido por $\rho(A)=\sup \{d(x, y): x, y \in A\}$ é chamado de diâmetro de A.

Proposição 1.9. Quaisquer que sejam $x \in E$ e $r \in] 0, \infty[$, tem-se:

(i) $\rho\left(D_{r}(x) \leq r\right.$;

(ii) $\rho\left(B_{r}(x) \leq r\right.$.

Definição 1.10. Seja $A \in E$ e $y \notin A$. Define-se a distância de y a $A$, e escreve-se $d(y, A)$, pela expressão

$$
d(y, A)=\inf \{d(y, x): x \in A\}
$$

Decorre dessa definição que $d(y, A) \geq 0$.

Proposição 1.11. Se $y \notin D_{r}$, então $d\left(y, D_{r}\right)=d(x, y)>0$, qualquer que seja $x \in D_{r}$.

\subsection{Números p-ádicos}

É sabido pela aritmética que todo número inteiro $n, n \neq 0$ e $n \neq \pm 1$, é igual ao produto finito de potências de números primos. Assim, quaisquer 
que sejam os números inteiros $n$ e $p, \operatorname{com} n$ não nulo e $p>0$ primo, existe $k \in \mathbb{Z}$ tal que $n=k \cdot p^{s}$, sendo $s$ é o maior número natural tal que $p^{s}$ divide $n$. É claro que se $p$ não divide $n$, então $s=0$.

Em toda esta seção consideraremos o número primo $p$ fixado.

Definição 1.12. Define-se a aplicação $\overline{\text { ord }}_{p}: \mathbb{Z} \rightarrow \mathbb{N} \cup\{\infty\}$ pela igualdade

$$
\overline{\operatorname{ord}}_{p}(n)=\left\{\begin{array}{lll}
s & \text {, se } & n=k \cdot p^{s} \\
\infty & \text {, se } & n=0
\end{array}\right.
$$

na qual $s$ é o maior número natural tal que $p^{s}$ divide $n$.

A função $\overline{\operatorname{ord}}_{p}$ está bem definida e, se definirmos $s+\infty=\infty+s=\infty$ para todo número natural $s$, a propriedade

$$
\overline{\operatorname{ord}}_{p}(m \cdot n)=\overline{\operatorname{ord}}_{p}(m)+\overline{\operatorname{ord}}_{p}(n)
$$

torna-se válida

Como veremos a seguir, essa definição pode ser estendida para o conjunto dos números racionais.

Definição 1.13. Define-se a aplicação ord $_{p}: \mathbb{Q} \rightarrow \mathbb{Z} \cup\{\infty\}$ por

$$
\operatorname{ord}_{p}(x)= \begin{cases}\overline{\operatorname{ord}}_{p}(a)-\overline{\operatorname{ord}}_{p}(b) & \text {, se } x=\frac{a}{b} \\ \infty & \text {, se } x=0\end{cases}
$$

Observações 1.14. A aplicação $\operatorname{ord}_{p}$ goza das propriedades:

(a) Para todo número inteiro $n$ tem-se $\operatorname{ord}_{p}(n)=\overline{\operatorname{ord}}_{p}(n)$.

(b) Essa definição independe da fração que expressa $x$.

(c) $\operatorname{ord}_{p}(x \cdot y)=\operatorname{ord}_{p}(x)+\operatorname{ord}_{p}(y)$

Definição 1.15. Uma função $|\cdot|: \mathbb{Q} \rightarrow[0, \infty[$ é chamada de valor absoluto em $\mathbb{Q}$ se, quaisquer que sejam $x, y \in \mathbb{Q}$, estão satisfeitas as condições: 
(i) $|x|=0$ se e somente se $x=0$;

(ii) $|x \cdot y|=|x| \cdot|y|$;

(iii) $|x+y| \leq|x|+|y|$.

Com essa definição às mãos verifica-se facilmente que a função $|\cdot|_{p}$, definida abaixo, é um valor absoluto em $\mathbb{Q}$,

Definição 1.16. Para todo $x \in \mathbb{Q}$, define-se

$$
|x|_{p}= \begin{cases}0 & , \mathrm{se} x=0 \\ p^{-\operatorname{ord}_{p}(x)} & , \mathrm{se} x \neq 0\end{cases}
$$

Observações 1.17. De 1.16 seguem-se as propriedades:

(a) $\left|p^{n}\right|_{p}=p^{-n}$,para todo $n \in \mathbb{N}$;

(b) $|x|_{p}=|-x|_{p} \geq 0$, para todo $x \in \mathbb{Q}$;

(c) $|x|_{p} \neq 0$ se $x \neq 0$;

(d) $|1|_{p}=p^{0}=1$;

(e) $\quad a$ e $b$ não são divisíveis por $p$, então $\left|\frac{a}{b}\right|_{p}=p^{0}=1$;

(f) $|x+y|_{p} \leqslant \max \left\{|x|_{p},|y|_{p}\right\}$;

(g) O par $\left(\mathbb{Q},|\cdot|_{p}\right)$ é um espaço ultramétrico.

Notações 1.18. Denota-se por $\mathbb{Q}_{p}$ o completado de $\mathbb{Q}$ com a métrica $|\cdot|_{p}$. Verifica-se que $\mathbb{Q}_{p}$ é o conjunto das séries formais $\sum_{k=m}^{\infty} a_{k} p^{k}$ na qual $m \in \mathbb{Z}$ e $0 \leq a_{k} \leq p-1$, para todo $k \geq m$. Se $a_{m} \neq 0$, então a série formal é única para cada $x \in \mathbb{Q}_{p}$. Com as operações usuais de séries podemos mostrar que $\mathbb{Q}_{p}$ é um corpo completo em relação ao valor absoluto $\left.\right|_{p}$. Seguindo a notação usual denotaremos por $Z_{p}$ o subconjunto de $\mathbb{Q}_{p}$ constituído por todos os números da forma $\sum_{k=0}^{\infty} a_{k} p^{k}$ - anel dos inteiros p-ádicos. 
Proposição 1.19. Para todo $x \in \mathbb{Q}_{p}$, com $x=\sum_{k=m}^{\infty} a_{k} p^{k}$, com $a_{m} \neq 0$, tem-se $|x|_{p}=p^{-m}$.

Segue-se dessa proposição que $\mathbb{Z}_{p}=D_{1}(0)$

Proposição 1.20. $\mathbb{Z}_{p}$ é compacto.

Proposição 1.21. Para todo $x \in \mathbb{Q}_{p}$ e todo $r \geq 0, D_{r}(x)$ é compacto.

Proposição 1.22. Todo disco ou toda bola aberta, de raio não nulo, é, simultaneamente, um conjunto aberto e fechado.

Corolário 1.23. Sejam $D_{1}, \ldots, D_{n}$ discos contidos em $D_{r}(x)$, dois a dois disjuntos. Então existem discos $F_{1}, \ldots, F_{m}$, contidos em $D_{r}(x)$, dois a dois disjuntos, satisfazendo $D_{r}(x)=\left(\bigcup_{i=1}^{n} D_{i}\right) \cup\left(\bigcup_{j=1}^{m} F_{m}\right)$.

Prova. Por (1.22), cada $D_{i}$ é um conjunto aberto. Para cada $y \notin \bigcup_{i=1}^{n} D_{i}$ consideremos o disco de centro $y$ e raio $r_{y}=\min \left\{d\left(y, D_{i}\right): 1 \leq i \leq n\right\}$. Por (1.11), $r_{y}>0$, para todo $y$. Segue-se que a coleção

$$
D_{1}, \ldots, D_{n}, D_{r_{y}}(y)\left(y \notin \bigcup_{i=1}^{n} D_{i}\right)
$$

é uma cobertura de $D_{r}(x)$ por abertos. A compacidade de $D_{r}(x)$ e (1.7) acarretam a existência de $y_{1}, \ldots, y_{m}$ tais que

$$
D_{r}(x)=D_{1} \bigcup \ldots \bigcup D_{n} \bigcup D_{r_{y_{1}}}\left(y_{1}\right) \bigcup \ldots \bigcup D_{r_{y_{m}}}\left(y_{m}\right)
$$

Fazendo, para todo $1 \leq j \leq m, F_{j}=D_{r_{u_{j}}}\left(y_{j}\right)$, a prova está completa.

Proposição 1.24. Para todo $x \in \mathbb{Q}_{p}$, e todo $r>0$, existe $n \in \mathbb{Z}$ tal que

(i) $B_{r}(x)=D_{p^{n}}(x)$;

(ii) $D_{r}(x)=D_{p^{n}}(x)$. 
Observação 1.25. À vista desta proposição somente são de interesse os discos cujos raios são da forma $p^{n}$, no qual $n$ é um número intciro. Assumiremos, a menos de citação em contrário, que todo disco de raio $r$ teremos $r \in\left\{p^{n}: n \in \mathbb{Z}\right\}$.

Corolário 1.26. Quaisquer que $\operatorname{sejam} x \in \mathbb{Q}_{p}$ e $n \in \mathbb{Z}$ tem-se $B_{p^{-n}}(x)=D_{p^{-n+1}}$ é compacto.

Proposição 1.27. O número de discos de raio $p^{-n}$ que cobrem $\mathbb{Z}_{p}$ é $p^{n}$.

Corolário 1.28. Sejam $m, n \in \mathbb{Z}$, com $m>n$, e seja $x \in \mathbb{Q}_{p}$. Então existem $p^{m-n}$ discos de raio $p^{-m}$ que cobrem $D_{p^{-n}}(x)$.

Teorema 1.29. Sejam $D, D_{1}, \ldots, D_{n}$ discos de raios $r, r_{1}, \ldots, r_{n}$, respectivamente, no qual cada $r_{i}$ é um número da forma $p^{\alpha}, \operatorname{com} \alpha \in \mathbb{Z}$. Se $\bigcup_{i=1}^{n} D_{i}=D$ e $D_{i} \cap D_{j}=\emptyset$, para todo $i \neq j$, então $r=r_{1}+\ldots+r_{n}$.

Prova. De $D_{i} \subset D$, segue-se que $r \geq r_{i}$, para todo $1 \leq i \leq n$. Inicialmente admitamos que $r_{1}=\ldots=r_{n}$. Por (1.28), $D$ é coberto por $\frac{r}{r_{n}}$ discos de raio $r_{n}$. Utilizando a hipótese, vem $\frac{r}{r_{n}}=n \mathrm{e}$, portanto, $r=n \cdot r_{n}$, verificando a conclusão para o caso de todos os discos terem o mesmo raio.

Suponhamos que $r_{1} \geq \ldots \geq r_{n}$. Então, por (1.28) $D$ é coberto por $\frac{r}{r_{n}}$ discos de raios $r_{n}$ e, para cada $1 \leq i \leq n$ o disco $D_{i}$ é coberto por $\frac{r_{i}}{r_{n}}$ discos de raios $r_{n}$. Como os discos $D_{1}, \ldots, \mathcal{D}_{n}$ são dois a dois disjuntos, então fracrr $_{n}=\frac{r_{1}}{r_{n}}+\ldots+\frac{r_{n}}{r_{n}}$ ou $r=r_{1}+\ldots+r_{n}$.

Teorema 1.30. Seja $A$ um subconjunto aberto de $D$. Então existe uma seqüência $E_{1}, \ldots, E_{n}, \ldots$ de discos contidos em $D$, dois a dois disjuntos, tais que $A=\bigcup_{n \geq 1} E_{n}$. 
Prova. Dado $x \in A$, seja $\Gamma$ o conjunto dos discos $E$ que satisfazem a condição $x \in E \subset A$. Por (1.22), $\Gamma$ é não vazio. Afirmamos que existe um disco $E_{x} \subset \Gamma$ tal que $E_{x}=\bigcup_{E \in \Gamma} E$

De fato, de $d(x, \complement A) \leq 1$, segue-se que existe

$$
s=\max \left\{z \in \mathbb{Z}: p^{n}<d(x, \complement A)\right\}
$$

Tomemos $E_{x}=D_{p^{*}}\left(x\right.$. É imediato que $x \in E_{x} \subset A$, logo

$$
E_{x} \subset \bigcup_{E \in \Gamma} E
$$

Por outro lado,dado $y \in \bigcup_{E \in \Gamma} E$ temos $y \in D_{p^{n}}(x)$, para algum $n \in \mathbb{Z}$. Como $p^{n} \leq d(x, \complement A)$, então $n \leq s$ e, portanto, $y \in E_{x}$; mostrando que

$$
\bigcup_{E \in \Gamma} E=E_{x}
$$

De (1) e (2) segue a igualdade

$$
E_{x}=\bigcup_{E \in \Gamma} E
$$

E, dessa,

$$
A=\bigcup_{x \in A} E_{x}
$$

Por (1.5), dados $x, y \in A$ temos $E_{x}=E_{y}$ ou $E_{x} \cap E_{y}=\emptyset$. Seja $B \subset A$ o conjunto dos $x \in A$ tais os discos $E_{x}$ são dois a dois disjuntos. Logo $A=\bigcup_{x \in B} E_{x}$.

Provemos que $B$ é enumerável.

De $\mathbb{Q}$ é denso em $\mathbb{Q}_{p}$ segue-se que para cada $x \in B$ existe um número racional $r(x) \in E_{x}$. Assim a função $f: B \rightarrow \mathbb{Q}$ definida por $f(x)=r(x)$ está bem definida e é injetora, pois se $r(x)=r(y)$ então $r(y) \in E_{x}$, contrariando o fato de que $E_{y} \cap E_{x}=\emptyset$, quaisquer que sejam $x, y \in B$. Logo $A=\bigcup_{n \geq 1} E_{n}$.

Para cada $x \in \mathbb{Q}_{p}$ e $A \subset \mathbb{Q}_{p}$, denotamos por $x+A$ o subconjunto de $\mathbb{Q}_{p}$ definido por

$$
x+A=\left\{z \in \mathbb{Q}_{p}: z=x+y, \text { para algum } y \in A\right\}
$$


Proposição 1.31. Sejam $F_{s}$ e $D_{r}$ discos contido em $\mathbb{Q}_{p}$, $\operatorname{com} F_{s} \subset D_{r}$.

Então, para todo $x \in \mathbb{Q}_{p}$, tem-se:

(i) $\quad\left(x+F_{s}\right) \subset D_{r}$, se $|x|_{p} \leq r$;

(ii) $\left(x+F_{s}\right) \cap D_{r}=\emptyset$, se $|x|_{p}>r$;

(iii) $\left(x+F_{s}\right)$ é um disco de raio $s$.

Prova. Dado $z \in x+F_{s}$ existe $z_{1} \in F_{s}$ tal que $z=x+z_{1}$. Segue-se que $\left|z-z_{1}\right|_{p}=|x|_{p}$. Se $|x|_{p} \leq r$, como $z_{1} \in F_{s} \subset D_{r}$, temos $z \in D_{r}\left(z_{1}\right)=D_{r}$. Isso mostra que $x+F_{s} \subset D_{r}$.

Se $|x|_{p}>r$ então $\left|z-z_{1}\right|_{p}>r$ e $z \notin D_{r}(y)=\emptyset$. Isso implica que $x+F_{s} \cap D_{r}=\emptyset$. Com isso provamos (i) e (ii).

Seja $y \in x+F_{s}$. Dado $z \in x+F_{s}$ existe $z_{1} \in F_{s}$ tal que $z=x+z_{1}$. Logo $|z-y|_{p}=\left|x+z_{1}-y\right|_{p}=\left|z_{1}-(y-x)\right|_{p} \leq s$ uma vez que $z_{1},(y-x) \in F_{s}$. Isso mostra que

$$
x+F_{s} \subset D_{s}(y)
$$

Por outro lado se $w \in D_{s}(y)$, então $|(w-x)-(y-x)|_{p}=|w-y|_{p} \leq s$, mostrando que $w_{1}=w-x \in D_{s}(y-x)=F-s$, pois $y-x \in F_{s}$. Segue-se que $w=x+w_{1}$, com $w_{1} \in F_{s}$. Portanto

$$
D_{s}(y) \subset x+F_{s}
$$

De (1) e (2) vem $x+F_{s}=D_{s}(y)$. 


\section{Capítulo 2}

\section{Integral de Kurzweil-Henstock}

\subsection{Introdução}

Iniciamos esta seção introduzindo os conceitos e terminologia que nos auxiliarão na compreensão da definição da integral de Kurzweil-Henstock.

Relembrando a Proposição (1.6) designaremos um disco de centro $x$ e raio $r$ simplesmente por $D_{r}$ quando o seu centro não for fundamental no desenvolvimento que estivermos realizando. Também, relembrando que a imagem da métrica em $\mathbb{Q}_{p}$ é o conjunto $\left\{p^{n}: n \in \mathbb{Z}\right\} \cup\{0\}$ fica estabelecido, para não sermos repetitivos, que os raios das bolas abertas e discos serão sempre elementos desse conjunto e os respectivos raios são não nulos; as exceções serão enunciadas.

No desenvolvimento de toda a teoria consideraremos fixo o disco $D$ de raio $r>0$.

Definição 2.1. Uma divisão de $D$ é um conjunto finito de discos, $\left\{D_{r_{1}}, \ldots, D_{r_{n}}\right\}$, contidos em $D$, satisfazendo as seguintes condições, para cada $1 \leq i \leq n$ : 
(i) $\quad r_{i}=p^{n_{i}}$, com $n_{i} \in \mathbb{Z}$;

(ii) $D_{r_{i}} \cap D_{r_{j}}=\emptyset$, se $i \neq j$;

(iii) $\bigcup_{i=1}^{n} D_{r_{i}}=D$.

A afirmação de que existe pelo menos uma divisão em $D$ satisfazendo a definição acima é dada por (1.28).

Definição 2.2. Uma divisão marcada de $D$ é um conjunto finito de pares ordenados, $\mathcal{D}=\left\{\left(x_{1}, D_{r_{1}}\right), \ldots,\left(x_{n}, D_{r_{n}}\right)\right\}$, no qual $\left\{D_{r_{1}}, \ldots, \mathcal{D}_{r_{n}}\right\}$ é uma divisão de $D$ e os pontos $x_{1}, \ldots x_{n}$ verificam a condição $x_{i} \in D_{r_{i}} \subset D$, para todo $1 \leq i \leq n$. Os elementos $x_{1}, \ldots, x_{n}$ são as marcas da divisão.

Definição 2.3. (i) Seja $\Lambda$ o conjunto de todas as bolas abertas de raio finito, contidas em $\mathbb{Q}_{p}$. Toda aplicação $\delta$ que associa a cada ponto $x \in D$ o elemento $\delta(x) \in \Lambda$, satisfazendo a condição $x \in \delta(x)$, é chamada de gauge em $D$.

(ii) Sejam $\delta$ um gauge e $\mathcal{D}=\left\{\left(x_{1}, D_{r_{1}}\right), \ldots,\left(x_{n}, D_{r_{n}}\right)\right\}$ uma divisão marcada de $D$. Dizemos que $\mathcal{D}$ é uma divisão marcada $\delta$-fina de $D$, e denotare$\operatorname{mos} \mathcal{D}<<\delta$, se $x_{i} \in D_{r_{i}} \subset \delta\left(x_{i}\right)$, para todo $1 \leq i \leq n$.

É claro que dada uma divisão marcada de $D$ é possível encontrar um gauge tal que essa divisão marcada seja $\delta$-fina. A recíproca dessa afirmação não é de todo evidente, entretanto ela é válida. Essa é a próxima proposição.

Proposição 2.4. Seja $\delta$ um gauge em $D$. Então, existe em $D$ uma divisão marcada $\delta$-fina, $\mathcal{D}=\left\{\left(x_{1}, D_{r_{1}}\right), \ldots,\left(x_{n}, D_{r_{n}}\right)\right\}$.

Prova. Seja $\Gamma=(\delta(x))_{x \in D}$ uma cobertura de D por abertos. A compacidade de $D$ implica a existência de $x_{1}, \ldots, x_{m} \in D$ satisfazendo

$$
D \subset \bigcup_{i=1}^{m} \delta\left(x_{i}\right) \text {. }
$$


Por (1.24), existem

$$
r_{1}, \ldots r_{m} \in\left\{p^{k}: k \in \mathbb{Z}\right\},
$$

satisfazendo

$$
D_{r_{i}}=\delta\left(x_{i}\right), \text { para todo } 1 \leq i \leq m .
$$

Lembrando (1.5) podemos assumir $\delta\left(x_{i}\right) \cap \delta\left(x_{j}\right)=\emptyset$, se $i \neq j$. Logo

$$
D_{r_{i}} \cap D_{r_{j}}=\emptyset, \text { se } i \neq j
$$

De (1) e (3) vem:

$$
D \subset \bigcup_{i=1}^{m} D_{r_{i}}
$$

Relembrando (1.7.i), existe $1 \leq n \leq m$, tal que

$$
D=\bigcup_{i=1}^{n} D_{r_{i}} .
$$

Os itens (2),(4) e (6) mostram-nos que as condições da definição (2.1) estão satisfeitas, e (3) mostra que a divisão é $\delta$-fina, completando a prova do teorema.

Definição 2.5. Sejam $f: D \rightarrow \mathbb{R}$ uma função e $\mathcal{D}=\left\{\left(x_{1}, D_{r_{1}}\right), \ldots,\left(x_{n}, D_{r_{n}}\right)\right\}$ uma divisão marcada de $D$. A soma de Riemann de $f$ em relação a $\mathcal{D}$, $S(f, \mathcal{D})$, é definida por $S(f, \mathcal{D})=\sum_{i=1}^{n} f\left(x_{i}\right) \cdot r_{i}$.

As ferramentas necessárias para a definição da integral de KurzweilHenstock estão disponíveis. Definamos essa integral.

Definição 2.6. Diz-se que uma função $f: D \rightarrow \mathbb{R}$ é Kurzweil-Henstock integrável em $D$ se existe um número real $I$ satisfazendo a condição: para cada $\varepsilon>0$ existe um gauge $\delta: D \rightarrow \Lambda$ tal que para toda $\mathcal{D}<<\delta$ tem-se $|S(f, \mathcal{D})-I|<\varepsilon$. 
Teorema 2.7. Se $f$ é uma função Kurzweil-Henstock integrável $\mathrm{cm} D$, então o elemento $I \in \mathbb{R}$ que satisfáz a definição acima é único.

Prova. Dado $\varepsilon>0$, sejam $I$ e $J$ números reais, $\delta_{1}$ e $\delta_{2}$ gauges definidos em $D$ tais que quaisquer que sejam $\mathcal{D}_{1}<<\delta_{1}$ e $\mathcal{D}_{2}<<\delta_{2}$ são válidas as desigualdades

$$
\left|S\left(f, \mathcal{D}_{1}\right)-I\right|<\frac{\varepsilon}{2} \quad \text { e } \quad\left|S\left(f, \mathcal{D}_{2}\right)-J\right|<\frac{\varepsilon}{2}
$$

Definamos $\delta(x)=\delta_{1}(x) \cap \delta_{2}(x)$, para todo $x \in D$. É claro que $\delta$ é um gauge em $D$ e que toda divisão $\mathcal{D}<<\delta$ é simultaneamente $\delta_{1}$ e $\delta_{2}$-finas. Portanto, ambas as desigualdades de (1) são válidas para $\mathcal{D}$. Segue-se

$$
|I-J| \leq|I-S(f, \mathcal{D})+S(f, \mathcal{D})-J| \leq|I-S(f, \mathcal{D})|+|S(f, \mathcal{D})-J|<\varepsilon
$$

e da arbitrariedade de $\varepsilon, I=J$.

Notações 2.8. O elemento $I \in \mathbb{R}$ que satisfaz a definição acima é chamado de integral de Kurzweil-Henstock de $f$ em $D$ e é denotado por $\int_{D} f$. Se a integral de $f$ em $D$ existe diremos que $f$ é integrável em $D$. O conjunto de todas as funções integráveis em $D$ será denotado por $\mathbb{K} \mathbb{H}(D)$.

Exemplo 2.9. (Uma função constante é integrável) Seja $f(x)=k, k \in \mathbb{R}$, para todo $x \in D$. Então $f \in \mathbb{K} \mathbb{H}(D)$ e $\int_{D} f=k r$

Prova. De fato, sejam $\varepsilon>0$ e $\delta$ um gauge arbitrário. Então, qualquer que seja $\mathcal{D}=\left\{\left(x_{1}, D_{r_{1}}\right), \ldots,\left(x_{n}, D_{r_{n}}\right)\right\}<<\delta$ e, lembrando o teorema (1.29), temos

$$
\left|\sum_{i=1}^{n} f\left(x_{i}\right) \cdot r_{i}-k r\right|=\left|\sum_{i=1}^{n} k \cdot r_{i}-k r\right|=\left|k \cdot\left[\sum_{i=1}^{n} r_{i}-r\right]\right|=0 .
$$

Decorrem desse exemplo: $\int_{\mathbb{Z}_{p}} \chi_{\mathbb{Z}_{p}}=1 \mathrm{c} \int_{D} \chi_{D}=r$. 
Exemplo 2.10. Mostremos que dado um disco $D_{s} \subset D$, a função $\chi_{D_{s}}$ é integrável em $D$ e $\int_{D} \chi_{D_{s}}=s$.

Sejam $\varepsilon>0, \delta$ um gauge arbitrário e $\mathcal{D}=\left\{\left(x_{1}, D_{r_{1}}\right), \ldots,\left(x_{n}, D_{r_{n}}\right)\right\}<<\delta$.

De $D_{s} \subset \bigcup_{i=1}^{n} D_{r_{i}}$ segue-se, por (1.7) que existem $i(1), \ldots, i(m)$, tais que

$$
D_{s}=\bigcup_{j=1}^{m} D_{r_{i}(j)}
$$

e, por (1.29)

$$
\sum_{j=1}^{m} r_{i(j)}=s
$$

Logo,

$$
\left|\sum_{i=1}^{n} \chi_{D_{s}}\left(x_{i}\right) \cdot r_{i}-s\right|=\left|\sum_{j=1}^{m} \chi_{D_{s}}\left(x_{i(j)}\right) \cdot r_{i(j)}-s\right|=|s-s|=0<\varepsilon,
$$

verificando a afirmação. $\diamond$

Exemplo 2.11. Seja $x=\sum_{k=0}^{\infty} a_{k} \cdot 3^{k} \in \mathbb{Z}_{3}$. Na expansão triádica de $x$ seja $j=\min \left\{k \geq 0: a_{k}=2\right\}$.

Isso posto, fixemos $n \geq 0$ e definamos a função

$$
f_{n}(x)= \begin{cases}\frac{(-1)^{j}}{j+1} \cdot \frac{3^{j+1}}{2^{j}} & , \text { se } j \leq n \\ 0 & , \text { nos demais pontos. }\end{cases}
$$

Mostremos que $f_{n}$ é integrável e $\int_{D} f_{n}=\sum_{j=0}^{n} \frac{(-1)^{j}}{j+1}$.

Dado $\varepsilon>0$ podemos assumir, sem perda de generalidade, que $\varepsilon<1$.

Seja $A=\left\{x=a_{0} \cdot 3^{0}+\ldots+2 \cdot 3^{j}+a_{j+1} \cdot 3^{j+1}+\ldots \in \mathbb{Z}_{3}: j \leq n\right\}$ 
É fácil verificar que $A$ é dado pela reunião de todos os discos com centro em um ponto da forma $x=a_{0} \cdot 3^{0}+\ldots+2 \cdot 3^{j}+a_{j+1} \cdot 3^{j+1}+\ldots$, com $j \leq n$ e raio $3^{-(j+1)}$. Além disso também podemos provar que:

$$
D_{3^{-\left(j_{1}+1\right)}}\left(\sum_{\substack{k=0 \\ j_{1} \leq n}}^{\infty} a_{k} \cdot 3^{k}\right) \cap D_{3^{-\left(j_{2}+1\right)}}\left(\sum_{\substack{k=0 \\ j_{2} \leq n}}^{\infty} b_{k} \cdot 3^{k}\right)=\emptyset
$$

se $a_{k} \neq b_{k}$ para algum $k \leq \min \left\{j_{1}, j_{2}\right\}$. Mais ainda, também podemos verificar que se $y \notin A$, a bola aberta $B_{3^{-n}}(y)=D_{3^{-(n+1)}}$ não contém nenhum número tendo o algarismo 2 na posição $j \leq n$. De fato, se $z \in B_{3^{-n}}(y)$ e $z$ possui o algarismo 2 na posição $j \leq n$ então $3^{-n}>|z-x|_{p}=3^{-j} \geq 3^{-n}$, que é uma contradição.

Com esses dados às mãos definamos o gauge $\delta$ em $\mathbb{Z}_{3}$ por

$$
\delta(x)= \begin{cases}B_{3^{-j}}(x)=D_{3^{-(j+1)}}(x) & \text { se } x \in A ; \\ B_{3^{-n}}(x)=D_{3^{-(n+1)}}(x) & \text { se } x \notin A\end{cases}
$$

Para toda $\mathcal{D}=\left\{\left(x_{i}, D_{r_{i}}\right): i=1, \ldots, m\right\}<<\delta$ temos: se $x_{i} \notin A$ então $f_{n}\left(x_{i}\right)=0$. Logo a soma de Riemann de $f_{n}$ reduz-se aos pontos pertencentes a $A$. Pelo exposto acima os discos de raio $3^{-(j+1)}$, centrados em pontos com $j \leq n$ somente podem ser cobertos por discos centrados em pontos da mesma forma e a soma dos raios desses discos, conforme (1.29) é $3^{-(j+1)}$. Por outro lado a quantidade de discos, dois a dois disjuntos, centrados em pontos da forma $a_{0} \cdot 3^{0}+\ldots+2 \cdot 3^{j}+a_{j+1} \cdot 3^{j+1}+\ldots$ é $2^{j}$. Segue-se:

$$
\sum_{j=0}^{n} \sum_{j \leq n} f\left(x_{i}\right) r_{i}=\sum_{j=0}^{n} 2^{j} \frac{(-1)^{j}}{j+1} \cdot \frac{3^{j+1}}{2^{j}} \cdot 3^{-(j+1)}=\sum_{j=0}^{n} \frac{(-1)^{j}}{j+1}
$$


Logo, para todo $\varepsilon>0$, tomamos $\delta$ o gauge acima. Assim, para toda $\mathcal{D}<<\delta$ temos

$$
\left|S\left(f_{n}, \mathcal{D}\right)-\sum_{j=0}^{n} \frac{(-1)^{j}}{j+1}\right|=0<\varepsilon
$$

Portanto $f_{n}$ é integrável e $\int_{D} f_{n}=\sum_{j=0}^{n} \frac{(-1)^{j}}{j+1}$.

\subsection{A integral como funcional linear}

O teorema a seguir mostrará que $\mathbb{K} \mathbb{H}(D)$, com a definição usual de soma e produto por um escalar, é um espaço vetorial sobre o corpo dos números reais. Também verificaremos que o funcional $f \rightarrow \int_{D} f$ é linear e positivo.

Teorema 2.12. (linearidade e positividade). Sejam $f$ e $g$ integráveis em D. Então,

(i) $f+g$ é integrável em $D$ e $\int_{D}(f+g)=\int_{D} f+\int_{D} g$;

(ii) af é integrável em $D$, para todo $a \in \mathbb{R}$, e $\int_{D}(a f)=a \cdot \int_{D} f$;

(iii) se $f \geq 0$, para todo $x \in D$, então $\int_{D} f \geq 0$.

Prova. Decorre da hipótese que dado $\varepsilon>0$ existem gauges $\delta_{1}$ e $\delta_{2}$ tais que, quaisquer que sejam as divisões $\mathcal{D}_{1}<<\delta_{1}$ e $\mathcal{D}_{2}<<\delta_{2}$, tem-se:

$$
\left|S\left(f, \mathcal{D}_{1}\right)-\int_{D} f\right|<\frac{\varepsilon}{2} \quad \text { e } \quad\left|S\left(g, \mathcal{D}_{2}\right)-\int_{D} g\right|<\frac{\varepsilon}{2} .
$$

Definamos o gauge $\delta(x)=\delta_{1}(x) \cap \delta_{2}(x)$, para todo $x \in D$. Logo, qualquer que seja a divisão $\mathcal{D}<<\delta$ temos que $\mathcal{D}<<\delta_{1}$ e $\mathcal{D}<<\delta_{2}$. Portanto,

$$
\begin{aligned}
\left|S(f+g, \mathcal{D})-\left[\int_{D} f+\int_{D} g\right]\right| & =\left|S(f, \mathcal{D})-\int_{D} f+S(g, \mathcal{D})-\int_{D} g\right| \\
& \leq\left|S(f, \mathcal{D})-\int_{D} f\right|+\left|S(g, \mathcal{D})-\int_{D} g\right| \\
& \leq \frac{\varepsilon}{2}+\frac{\varepsilon}{2}=\varepsilon .
\end{aligned}
$$

mostrando que $f+g$ é integrável e $\int_{D}(f+g)=\int_{D} f+\int_{D} g$. Isso encerra a prova de (i). 
Para a prova de (ii) consideremos inicialmente $a=0$. Logo $a f=0$ e, por (2.9) a função é integrável e a igualdade em (ii) é válida.

Admitamos $a \neq 0$. Logo $|a| \neq 0$.

Da hipótese, dado $\varepsilon>0$ existe um gauge $\delta$ tal que para toda divisão $\mathcal{D}<<\delta$ tem-se

$$
\left|S(f, \mathcal{D})-\int_{D} f\right|<\frac{\varepsilon}{|a|}
$$

Segue-se que

$$
\begin{aligned}
\left|S(a f, \mathcal{D})-a \cdot \int_{D} f\right| & =\left|a \cdot\left[S(f, \mathcal{D})-\int_{D} f\right]\right| \\
& =|a| \cdot\left|S(f, \mathcal{D})-\int_{D} f\right| \\
& <|a| \cdot \frac{\varepsilon}{|a|}=\varepsilon .
\end{aligned}
$$

mostrando que af é integrável em $D$ e $\int_{D} a f=a \int_{D} f$, e encerrando a prova de (ii).

Para a prova de (iii) consideremos $f>0$. Então, para toda divisão marcada $\mathcal{D}$ tem-se $S(f, \mathcal{D}) \geq 0$. Da hipótese, dado $\varepsilon>0$ seja $\delta$ o gague que satisfaz a definição da integral. Portanto dada $\mathcal{D}<<\delta$ tem-se

$$
\left|S(f, \mathcal{D})-\int_{D} f\right|<\varepsilon \Rightarrow 0 \leq S(f, \mathcal{D})<\int_{D} f+\varepsilon,
$$

para todo $\varepsilon>0$. A conclusão segue da arbitrariedade de $\varepsilon$.

Corolário 2.13. Se $f, g: D \rightarrow \mathbb{R}$ são funções integráveis em $D$ e $f(x) \leq g(x)$, para todo $x \in D$, então $\int_{D} f \leq \int_{D} g$.

Prova. Segue de $\int_{D}(f-g) \geq 0$, aplicando o teorema anterior.

Corolário 2.14. Se $f$ e $|f|$ são integráveis em $D$, então $\left|\int_{D} f\right| \leq \int_{D}|f|$.

Prova. Segue de $-|f| \leq f \leq|f|$ e aplicando o corolário anterior.

\subsection{O Critério de Cauchy}

O próximo teorema estabclece o critério de Cauchy para a integral de Kurzweil-Henstock. Essa condição apresenta as mesmas vantagens oferecidas pelo critério de Cauchy para as seqüências, isto é, permite-nos concluir se uma função é integrável sem a necessidade de conhecermos o seu valor. 
Teorema 2.15. (Critério de Cauchy) Seja $f: D \rightarrow \mathbb{R}$ uma função. A função $f$ é integrável em $D$ se e somente se, dado $\varepsilon>0$, existe um gauge $\delta$ tal que, quaisquer que sejam as divisões marcadas $\delta$-finas $\mathcal{D}_{1}$ e $\mathcal{D}_{2}$, tem-se $\left|S\left(f, \mathcal{D}_{1}\right)-S\left(f, \mathcal{D}_{2}\right)\right|<\varepsilon$.

Prova. Necessária. De fato, dado $\varepsilon>0$, sejam $\delta$ o gauge e $\mathcal{D}_{1}$ e $\mathcal{D}_{2}$, divisões marcadas $\delta$-finas de $D$, satisfazendo

$$
\left|S\left(f, \mathcal{D}_{1}\right)-\int_{D} f\right|<\frac{\varepsilon}{2} \quad \text { e } \quad\left|S\left(f, \mathcal{D}_{2}\right)-\int_{D} f\right|<\frac{\varepsilon}{2} .
$$

Segue-se

$$
\left|S\left(f, \mathcal{D}_{1}\right)-S\left(f, \mathcal{D}_{2}\right)\right| \leq\left|S\left(f, \mathcal{D}_{1}\right)-\int_{D} f\right|+\left|S\left(f, \mathcal{D}_{2}\right)-\int_{D} f\right|<\varepsilon .
$$

verificando a condição necessária.

Suficiente. Para cada $n>1$, tomemos $\delta_{n}$, gauge sobre $D$ tal que quaisquer que sejam as divisões marcada $\delta_{n}$-finas, $\mathcal{D}_{1}$ e $\mathcal{D}_{2}$ tenhamos

$$
\left|S\left(f, \mathcal{D}_{1}\right)-S\left(f, \mathcal{D}_{2}\right)\right|<\frac{1}{n}
$$

Sem perda de generalidade, podemos assumir que $\delta_{n+1}(x) \subset \delta_{n}(x)$, para todo $x \in D$ e todo $n \geq 1$.

Designando por $\Gamma_{n}=\left\{S(f, \mathcal{D}): \mathcal{D}<<\delta_{n}\right\}$ e escolhendo, para cada $n \geq 1$, $\mathcal{D}_{n}$ satisfazendo $S\left(f, \mathcal{D}_{n}\right) \in \Gamma_{n}$ temos,

$$
\left|S\left(f, \mathcal{D}_{m}\right)-S\left(f, \mathcal{D}_{n}\right)\right|<\frac{1}{n}
$$

para todo $m>n$.

Portanto a seqüência $\left(S\left(f, \mathcal{D}_{n}\right)\right)_{n \geq 1}$ é de Cauchy em $\mathbb{R}$.

A completude de $\mathbb{R}$ acarreta a existência de $I \in \mathbb{R}$ tal que, para um dado $\varepsilon>0$ existe $n_{0} \geq 1$ satisfazendo $\left|I-S\left(f, \mathcal{D}_{n}\right)\right|<\frac{\varepsilon}{2}$, para todo $n \geq n_{0}$.

Escolhendo $m \geq \max \left\{n_{0}, \frac{2}{\varepsilon}\right\}$, segue-se, para toda $\mathcal{D}<<\delta_{m}$,

$$
\begin{aligned}
|S(f, \mathcal{D})-I| & =\left|S(f, \mathcal{D})-S\left(f, \mathcal{D}_{m}\right)+S\left(f, \mathcal{D}_{m}\right)-I\right| \\
& \leq\left|S(f, \mathcal{D})-S\left(f, \mathcal{D}_{m}\right)\right|+\left|S\left(f, \mathcal{D}_{m}\right)-I\right| \\
& <\varepsilon
\end{aligned}
$$

mostrando que $f$ é integrável. 


\subsection{A integral como função de discos}

Teorema 2.16. Se $f$ é integrável em $D$ e $E$ é um disco contido em $D$, então

(i) $\quad f$ (restrita a $E$ ) é integrável em $E$;

(ii) $f \chi_{E}$ é integrável em $D$;

(iii) $\int_{D} f \chi_{E}=\int_{E} f$

Prova. (i) A compacidade de $D$ e usando(1.23), existem discos $F_{1}, \ldots, F_{n}$, dois a dois disjuntos, verificando $D=E \cup\left(\bigcup_{j=1}^{n} F_{n}\right)$.

Dado $\varepsilon>0$ temos, por hipótese, que existe um gauge $\delta$ em $D$ satisfazendo o critério de Cauchy.

Seja $\bar{\delta}$ um gauge em $E$ definido por $\bar{\delta}(x)=\delta(x)$, para todo $x \in E$, e sejam $\overline{\mathcal{D}_{1}}, \overline{\mathcal{D}_{2}}<<\bar{\delta}$.

Para cada $1 \leq j \leq n$, definamos o gauge $\delta^{j}$ em $F_{j}$ por $\delta^{j}(x)=\delta(x)$, para cada $x \in F_{j}$, e tomemos $\left(\right.$ em $F_{j}$ ) as divisões $\mathcal{D}^{j}<<\delta^{j}$

Segue-se que

$$
\mathcal{D}_{1}=\overline{\mathcal{D}_{1}} \cup \mathcal{D}^{1} \cup \ldots \cup D^{n} \text { e } \mathcal{D}_{2}=\overline{\mathcal{D}_{2}} \cup \mathcal{D}^{1} \cup \ldots \cup D^{n}
$$

são divisões marcadas $\delta$-finas de $D$. Portanto

$$
\begin{array}{r}
\left|S\left(f, \overline{\mathcal{D}_{1}}\right)-S\left(f, \overline{\mathcal{D}_{2}}\right)\right|=\left|S\left(f, \overline{\mathcal{D}_{1}}\right)+\sum_{k=1}^{n} S\left(f, \mathcal{D}^{k}\right)-\sum_{k=1}^{n} S\left(f, \mathcal{D}^{k}\right)-S\left(f, \overline{\mathcal{D}_{2}}\right)\right| \\
=\left|S\left(f, \overline{\mathcal{D}_{1}} \cup \mathcal{D}^{1} \cup \ldots \cup D^{n}\right)-S\left(f, \overline{\mathcal{D}_{2}} \cup \mathcal{D}^{1} \cup \ldots \cup D^{n}\right)\right| \\
=\left|S\left(f, \mathcal{D}_{1}\right)-S\left(f, \mathcal{D}_{2}\right)\right|<\varepsilon
\end{array}
$$

Pelo Critério de Cauchy, $f$ é integrável em $E$, provando (i).

As funções $f$ e $f \chi_{E}$ são iguais em $E$. Então, por (i), $f \chi_{E}$ é integrável em $E$ e $\int_{E} f \chi_{E}=\int_{E} f$.

Provemos que $f \chi_{E}$ é integrável em $D$.

Como $f \chi_{E}$ é integrável em $E$, dado $\varepsilon>0$ existe um gauge $\bar{\delta}$ tal que para toda $\mathcal{D}_{1}<<\bar{\delta}$ temos $\left|\int_{E} f-S\left(f, \mathcal{D}_{1}\right)\right|<\varepsilon$.

Definamos o gauge $\delta$ em $D$ por :

$$
\delta(x)= \begin{cases}\bar{\delta}(x) & \text { se } x \in E \\ F_{j} & \text { se } x \in D-E\end{cases}
$$


Agora, dada uma divisão

$$
\mathcal{D}=\left\{\left(x_{1}, E_{r_{1}}\right), \ldots,\left(x_{n}, E_{r_{n}}\right),\left(z_{1}, F_{s_{1}}\right), \ldots,\left(z_{m}, F_{s_{m}}\right)\right\}<<\delta,
$$

$\operatorname{com} x_{1}, \ldots, x_{n} \in E$ e $z_{1}, \ldots, z_{m} \in D-E$, vem,

$$
\begin{aligned}
& \left|S\left(f \chi_{E}, \mathcal{D}\right)-\int_{E} f\right|=\left|\sum_{i=1}^{n} f\left(x_{i}\right) r_{i}+\sum_{j=1}^{m} f\left(z_{j}\right) s_{j}-\int_{E} f\right| \\
& =\left|\sum_{i=1}^{n} f\left(x_{i}\right) r_{i}-\int_{E} f\right|<\varepsilon .
\end{aligned}
$$

provando (ii) e (iii).

Corolário 2.17. Se $\left\{D_{1}, \ldots, D_{n}\right\}$ é uma divisão de $D$ e $f$ é integrável em $D$, então $f$ é integrável em $D_{i}$, para cada $1 \leq i \leq n$ e $\int_{D} f=\sum_{i=1}^{n} \int_{D_{i}} f$.

Prova. Por (2.16) $f$ é integrável em cada um dos discos $D_{1}, \ldots, D_{n}$. Agora, como $f=\sum_{i=1}^{n} f \chi_{D_{i}}$ temos, usando (2.16) e (2.12.i),

$$
\int_{D} f=\int_{D} \sum_{i=1}^{n} f \chi_{D_{i}}=\sum_{i=1}^{n} \int_{D} f \chi_{D_{i}}=\sum_{i=1}^{n} \int_{D_{i}} f
$$

Exemplo 2.18. Segue-se dos teoremas acima e do exemplo (2.9) que se $E$ é um disco de raio $s$ contido em $D$, então $\int_{D} \chi_{E}=\int_{E} \chi_{E}=s \quad \diamond$

Teorema 2.19. Se $E$ é um disco contido em $D$ e $f$ é integrável em $E$, então a função $f \chi_{E}$, prolongamento de $f$ em $D$, é integrável em $D$ e $\int_{D} f \chi_{E}=\int_{E} f$.

Prova. Se $E=D$ nada a provar. Admitamos $E \neq D$.

Dado $\varepsilon>0$ seja $\bar{\delta}$ um gauge em $E$ tal que, para toda $\mathcal{D}_{1}<<\bar{\delta}$, tenhamos

$$
\left|S\left(f, \mathcal{D}_{1}\right)-\int_{E} f\right|<\varepsilon
$$

Por (1.23), sejam $F_{1}, \ldots, F_{m}$, dois a dois disjuntos, satisfazendo 


$$
D=E \cup F_{1} \cup \ldots \cup F_{m}
$$

Por (1.22), $F_{1}, \ldots, F_{m}$ são abertos. Assim podemos definir em $D$ o gauge

$$
\delta(x)= \begin{cases}\bar{\delta}(x) & \text {, se } x \in E ; \\ F_{j} & \text {, se } x \in F_{j}, 1 \leq j \leq m\end{cases}
$$

Logo, para toda $\mathcal{D}=\left\{\left(x_{1}, r_{1}, \ldots,\left(x_{n}, r_{n}\right)\right\}<<\delta\right.$ temos:

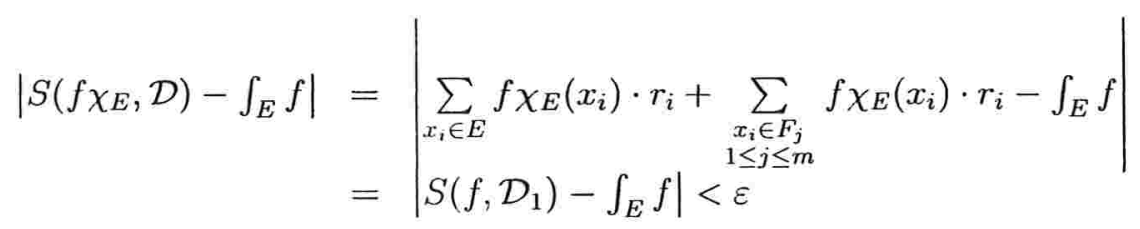

encerrando a prova do teorema.

Corolário 2.20. Se $D_{1}, \ldots, D_{n}$ são discos contidos em $D$, dois a dois disjuntos, tais que $\bigcup_{i=1}^{n} D_{i}=D$ e $f$ é integrável em $D_{i}$, para cada $1 \leq i \leq n$, então $f$ é integrável em $D$ e $\int_{D} f=\sum_{i=1}^{n} \int_{D_{i}} f$.

Prova. Para cada $x \in D$, temos $f(x)=\sum_{i=1}^{n}\left(f \chi_{D_{i}}\right)(x)$. Segue-se, por (2.12.i) que $f$ é integrável e

$$
\int_{D} f=\int_{D} \sum_{i=1}^{n}\left(f \chi_{D_{i}}\right)=\sum_{i=1}^{n} \int_{D} f \chi_{D_{i}}=\sum_{i=1}^{n} \int_{D_{i}} f
$$

encerrando a prova do teorema.

Teorema 2.21. Seja $f \geq 0$ integrável em $D$. Se $D_{1}, \ldots, D_{n}$ são discos contidos em $D$, dois a dois disjuntos, então $0 \leq \sum_{i=1}^{n} \int_{D_{i}} f \leq \int_{D} f$.

Prova. Por (1.23) existem discos $F_{1}, \ldots, F_{m}$ verificando

$$
D=D_{1} \bigcup \ldots \bigcup D_{n} \bigcup\left(\bigcup_{j=1}^{m} F_{j}\right) \text {. }
$$


Por (2.16) f é integrável em $D_{i}$, para cada $1 \leq i \leq n$, e em $F_{j}$, para cada $1 \leq j \leq m$. Além disso, por hipótese, $f \geq 0$, então por (2.12.iii), $\int_{D_{i}} f \geq 0 \mathrm{e}$ $\int_{F_{j}} f \geq 0$, para todo $i$ e todo $j$. Segue-se, pelo teorema anterior, que

$$
0 \leq \sum_{i=1}^{n} \int_{D_{i}} f \leq \sum_{i=1}^{n} \int_{D_{i}} f+\sum_{j=1}^{m} \int_{F_{j}} f=\int_{D} f
$$

completando a prova do teorema.

Definição 2.22. Sejam $E$ um subconjunto e $f$ uma função definida em $D$.

Se a função $f \chi_{E} \in \mathbb{K} \mathbb{H}(D)$ definimos $\int_{E} f=\int_{D} f \chi_{E}$.

\subsection{A integral e as funções contínuas}

Quando se estuda integrabilidade de funções, de imediato questiona-se a integrabilidade das funções contínuas. No que segue verificaremos que as mesmas são integráveis.

Lema 2.23. Seja $f: D \rightarrow \mathbb{R}$ e suponhamos que para cada $\varepsilon>0$ existam funções $g_{\varepsilon}, h_{\varepsilon} \rightarrow \mathbb{R}$ integráveis, satisfazendo as condições

(i) $g_{\varepsilon}(x) \leq f(x) \leq h_{\varepsilon}(x)$, para todo $x \in D$,

(ii) $\int_{D}\left(h_{\varepsilon}(x)-g_{\varepsilon}(x)\right)<\frac{\varepsilon}{2}$.

Então, f é integrável em $D$.

Prova. Da hipótese segue-se que dado $\varepsilon>0$ existem os gauges $\delta_{1}$ e $\delta_{2}$ tais que quaisquer que sejam as divisões $\mathcal{D}_{1}<<\delta_{1}$ e $D_{2}<<\delta_{2}$ tem-se

$$
\left|S\left(g_{\varepsilon}, \mathcal{D}_{1}\right)-\int_{D} g_{\varepsilon}\right|<\frac{\varepsilon}{4} \quad \text { e } \quad\left|S\left(h_{\varepsilon}, \mathcal{D}_{2}\right)-\int_{D} h_{\varepsilon}\right|<\frac{\varepsilon}{4} .
$$

Definamos, em $D$, o gauge $\delta(x)=\delta_{1}(x) \cap \delta_{2}(x)$, para todo $x$. Então, qualquer que seja $\mathcal{D}<<\delta$ temos que $\mathcal{D}$ é $\delta_{1}$ e $\delta_{2}$ - fina e, portanto,

$$
\int_{D} g_{\varepsilon}-\frac{\varepsilon}{4}<S\left(g_{\varepsilon}, \mathcal{D}\right) \leq S(f, \mathcal{D}) \leq S\left(h_{\varepsilon}, \mathcal{D}\right)<\int_{D} h_{\varepsilon}+\frac{\varepsilon}{4}
$$


Vem daí que, se $\mathcal{D}_{1}$ e $\mathcal{D}_{2}$ são divisões quaisquer de $D, \delta$-finas, então, usando a desigualdade acima,

$$
-\varepsilon<-\int_{D}\left(h_{\varepsilon}-g_{\varepsilon}\right)-\frac{\varepsilon}{2} \leq S\left(f, \mathcal{D}_{1}\right)-S\left(f, \mathcal{D}_{2}\right) \leq \int_{D}\left(h_{\varepsilon}-g_{\varepsilon}\right)+\frac{\varepsilon}{2}<\varepsilon
$$

mostrando que $\left|S\left(f, \mathcal{D}_{1}\right)-S\left(f, \mathcal{D}_{2}\right)\right|<\varepsilon$ e, portanto, $f$ é integrável pelo Critério de Cauchy.

Teorema 2.24. Se $f: D \rightarrow \mathbb{R}$ ć uma função contínua, então $f$ é integrável.

Prova. A função $f$ é contínua no compacto $D$, logo uniformemente contínua em $D$. Dado $\varepsilon>0$, e lembrando que o raio de $D$ é $r$, existe um $n \geq 0$ satisfazendo a condição

$$
|f(x)-f(y)|<\frac{\varepsilon}{r}, \quad \text { sempre que } \quad|x-y|_{p}<p^{-n} \leq r
$$

quaisquer que sejam $x, y \in D$.

Seja $t \geq n$ um número natural. Pelo corolário (1.28) existem $\lambda=p^{t} \cdot r$ discos, $E_{1}, \ldots, E_{\lambda}$, dois a dois disjuntos e de raio $p^{-t}$ satisfazendo $\bigcup_{j=1}^{\lambda} E_{\lambda}=D$. Seja $E_{j}$ um de tais discos e sejam

$$
m_{E_{j}}=\min \left\{f(x): x \in E_{j}\right\} \quad \text { e } \quad M_{E_{j}}=\max \left\{f(x): x \in E_{j}\right\}
$$

Pelo exemplo (2.9) as funções $m_{E_{j}} \chi_{E_{j}}$ e $M_{E_{j}} \chi_{E_{j}}$ são integráveis em $E_{j}$, para todo $1 \leq j \leq n$. Por $(2.20)$ as funções $g=\sum_{j=1}^{\lambda} m_{E_{j}} \chi_{E_{j}}$ e $h=\sum_{j=1}^{\lambda} M_{E_{j}} \chi_{E_{j}}$ são integráveis em $D$ com

$$
\int_{D} \sum_{j=1}^{\lambda} m_{E_{j}} \chi_{E_{j}}=\sum_{j=1}^{\lambda} m_{E_{j}} p^{-t} \text { e } \int_{D} \sum_{j=1}^{\lambda} M_{E_{j}} \chi_{E_{j}}=\sum_{j=1}^{\lambda} M_{E_{j}} p^{-t}
$$

Sejam $\delta_{1}$ e $\delta_{2}$ gauges definidos em $D$ tais que, quaisquer que sejam as divisões $\mathcal{D}_{1}<<\delta_{1}$ e $\mathcal{D}_{2}<<\delta_{2}$,

$$
\left|S\left(g, \mathcal{D}_{1}\right)-\sum_{j=1}^{\lambda} m_{E_{j}} p^{-t}\right|<\varepsilon \quad \text { c }\left|S\left(h, \mathcal{D}_{2}\right)-\sum_{j=1}^{\lambda} M_{E_{j}} p^{-t}\right|<\varepsilon
$$


Definamos o gauge $\delta(x)=\delta_{1}(x) \cap \delta_{2}(x)$, para todo $x \in D$. Então, se $\mathcal{D}_{1}=\left\{\left(x_{1}, D_{r_{1}}\right), \ldots,\left(x_{n}, D_{r_{n}}\right)\right\}$ e $\mathcal{D}_{2}=\left\{\left(y_{1}, D_{s_{1}}\right), \ldots,\left(y_{m}, D_{s_{m}}\right)\right\}$ são divisões marcadas $\delta$-finas, temos

$$
\begin{aligned}
& \left|S\left(f, \mathcal{D}_{1}\right)-S\left(f, \mathcal{D}_{2}\right)\right|=\left|\sum_{i=1}^{n} f\left(x_{i}\right) r_{i}-\sum_{k=1}^{m} f\left(y_{k}\right) s_{k}\right| \\
& \leq\left|\sum_{i=1}^{n} f(x) r_{i}-\sum_{j=1}^{\lambda} m_{E_{j}} p^{-t}\right|+\left|\sum_{j=1}^{\lambda} M_{E_{j}} p^{-t}-\sum_{k=1}^{m} f\left(y_{k}\right) s_{k}\right| \\
& +\left|\sum_{j=1}^{\lambda} M_{E_{j}} p^{-t}-\sum_{j=1}^{\lambda} m_{E_{j}} p^{-t}\right| \\
& <\varepsilon+\varepsilon+\left|p^{-t} \sum_{j=1}^{\lambda}\right| M_{E_{j}}-m_{E_{j}}||<2 \varepsilon+p^{-t} \cdot \lambda \cdot \frac{\varepsilon}{r}=3 \varepsilon
\end{aligned}
$$

provando que $f$ é integrável em $D$.

Teorema 2.25. Seja $f>0$ integrável em $D$. Se $g$ é contínua em $D$, então $f g$ é integrável em $D$.

Prova. De $f>0$ segue-se que $\int_{D} f>0$.

De $D$ compacto segue-se que $g$ é uniformemente contínua. Logo dado $\varepsilon>0$ existe um $n \in \mathbb{Z}$ tal que $p^{n}<r$ e quaisquer que sejam $x$ e $y$ em $D$, vale

$$
|x-y|_{p}<p^{n} \Rightarrow g(y)-\frac{\varepsilon}{2 \int_{D} f}<g(x)<g(y)+\frac{\varepsilon}{2 \int_{D} f}
$$

Por (1.28) existem $m=p^{-n} \cdot r$ discos de raio $p^{n}$ cobrindo $D$. Sejam $D_{1}, \ldots, D_{m}$ os discos que constituem essa divisão e sejam $x_{1}, \ldots, x_{m}$ satisfazendo $x_{i} \in D_{i}$ para cada $i$.

Definamos as funções

$$
\phi(x)=\sum_{i=1}^{m}\left(g\left(x_{i}\right)-\frac{\varepsilon}{2 \int_{D} f}\right) \cdot \chi_{D_{i}}(x)
$$

e

$$
\psi(x)=\sum_{i=1}^{m}\left(g\left(x_{i}\right)+\frac{\varepsilon}{2 \int_{D} f}\right) \cdot \chi_{D_{i}}(x)
$$


Segue-se de (1) que, para todo $x \in D$,

$$
\phi(x) \leq g(x) \leq \psi(x)
$$

e, como $f>0$, então

$$
f \phi \leq f g \leq f \psi
$$

para todo $x \in D$.

Por (2.10) $\chi_{D_{i}}$ é integrável em $D$, para cada $1 \leq i \leq m$; por (2.16.i) e (ii) $f \phi$ e $f \psi$ são integráveis em $D$ e, usando (2.17),

$$
\int_{D}(f \psi-f \phi)=\int_{D} \sum_{i=1}^{m} \frac{\varepsilon}{\int_{D} f} f \chi_{D_{i}}=\frac{\varepsilon}{\int_{D} f} \int_{D} f=\varepsilon
$$

Com (1) e (2) as hipóteses de (2.23) estão satisfeitas e $f g$ é integrável em $D$.

\subsection{O Lema de Henstock}

O Lema de Henstock afirma que se $\delta$ é um gauge em $D$ tal que qualquer divisão marcada $\delta$-fina nos dá uma boa aproximação da integral por somas de Riemann, então essa divisão marcada também é uma boa aproximação da integral por somas de Riemann para a reunião de discos finitos caracterizados pelo gauge $\delta$.

Iniciemos esta seção com uma definição

Definição 2.26. Seja $\delta$ um gauge em $D$. Uma coleção finita de pares

$$
\mathcal{D}=\left\{\left(x_{1}, D_{r_{1}}\right), \ldots,\left(x_{n}, D_{r_{n}}\right)\right\}
$$

satisfazendo:
(a) $x_{i} \in D_{r_{i}} \subset \delta\left(x_{i}\right)$, para todo $1 \leq i \leq n$;
(b) $D_{r_{i}} \cap D_{r_{j}}=\emptyset$, se $i \neq j$;
(c) $\bigcup_{i=1}^{n} D_{r_{i}} \subset D$. 
será chamada de divisão marcada parcial $\delta$-fina de $D$ e será denotada por $\mathcal{E}<<<\delta$. de $D$.

É claro que toda divisão marcada $\delta$-fina é uma divisão marcada parcial

Lema 2.27. (Lema de Henstock) Sejam $f$ integrável em $D$ e $\delta$ o gauge correspondente a um dado $\varepsilon>0$ na definição de integral. Então, qualquer que seja $\mathcal{D}=\left\{\left(x_{1}, D_{r_{1}}\right), \ldots,\left(x_{n}, D_{r_{n}}\right)\right\}<<<\delta$, as seguintes condições são válidas:
(i) $\left|\sum_{i=1}^{n}\left(f\left(x_{i}\right) r_{i}-\int_{D_{r_{i}}} f\right)\right| \leq \varepsilon$;
(ii) $\sum_{i=1}^{n}\left|f\left(x_{i}\right) r_{i}-\int_{D_{r_{i}}} f\right| \leq 2 \varepsilon$ :
(iii) $\sum_{i=1}^{n}|| f\left(x_{i}\right)\left|r_{i}-\right| \int_{D_{r_{i}}} f|| \leq 2 \varepsilon$;

Prova. Pelo Cor.(1.7), sejam $E_{1}, \ldots, E_{m}$ discos tais que

$$
D=\left(\bigcup_{i=1}^{n} D_{r_{i}}\right) \bigcup\left(\bigcup_{j=1}^{m} E_{j}\right)
$$

Por hipótese $f$ é integrável em $D$, logo integrável em cada $E_{j}$, para cada $1 \leq j \leq m$. Portanto, dado $\sigma>0$, existe, para cada $j$, um gauge $\delta_{j}$ tal que se $\mathcal{D}_{j}$ é uma divisão $\delta_{j}$-fina de $E_{j}$, então

$$
\left|S\left(f, \mathcal{D}_{j}\right)-\int_{E_{j}} f\right|<\frac{\sigma}{2^{m}} \quad \text { para cada } \quad 1 \leq j \leq m
$$

Definindo, em $D$, o gauge

$$
\bar{\delta}(x)=\left\{\begin{array}{llll}
\delta(x) & , \text { se } & x \in D_{r_{i}} & , 1 \leq i \leq n \\
\delta_{j}(x) & , \text { se } \quad x \in E_{j} & , 1 \leq j \leq m
\end{array}\right.
$$


temos que

$$
\mathcal{D}=\left\{\left(x_{1}, D_{r_{1}}\right), \ldots\left(x_{n}, D_{r_{n}}\right)\right\} \bigcup\left(\bigcup_{j=1}^{m} \mathcal{D}_{j}\right)<<\bar{\delta}
$$

e satisfaz

$$
\begin{aligned}
& \left|\sum_{i=1}^{n}\left(f\left(x_{i}\right) r_{i}-\int_{D_{r_{i}}} f\right)\right|<\left|\sum_{i=1}^{n}\left(f\left(x_{i}\right) r_{i}-\int_{D_{r_{i}}} f\right)\right|-\sum_{j=1}^{m} \frac{\sigma}{2^{m}}+\sigma \\
& <\left|\sum_{i=1}^{n}\left(f\left(x_{i}\right) r_{i}-\int_{D_{r_{i}}} f\right)\right|-\left|\sum_{j=1}^{m}\left(S\left(f, \mathcal{D}_{j}\right)-\int_{E_{j}} f\right)\right|+\sigma \\
& \leq\left|\sum_{i=1}^{n}\left(f\left(x_{i}\right) r_{i}-\int_{D_{r_{i}}} f\right)+\sum_{j=1}^{m}\left(S\left(f, \mathcal{D}_{j}\right)-\int_{E_{j}} f\right)\right|+\sigma \\
& =\left|S(f, \mathcal{D})-\int_{D} f\right|<\varepsilon+\sigma
\end{aligned}
$$

Como a desigualdade acima é válida para todo $\sigma>0$, vem

$$
\left|\sum_{i=1}^{n}\left(f\left(x_{i}\right) r_{i}-\int_{D_{r_{i}}} f\right)\right| \leq \varepsilon
$$

concluindo a prova de (i).

Para a prova de (ii), sejam A e B os conjuntos dos discos $D_{r_{i} \text { : }}$. $1 \leq i \leq n$, que satisfazem as relações

$$
f\left(x_{i}\right) r_{i}-\int_{D_{r_{i}}} f \geq 0 \quad \text { e } \quad f\left(x_{i}\right) r_{i}-\int_{D_{r_{i}}} f<0
$$

respectivamente.

Aplicando (i) a cada um dos conjuntos temos

$$
\left|\sum_{D_{r_{i}} \in A}\left(f\left(x_{i}\right) r_{i}-\int_{D_{r_{i}}} f\right)\right| \leq \varepsilon \quad \text { e } \quad\left|\sum_{D_{r_{i}} \in B}\left(f\left(x_{i}\right) r_{i}-\int_{D_{r_{i}}} f\right)\right| \leq \varepsilon .
$$


Segue

$$
\begin{aligned}
\sum_{i=1}^{n}\left|\left(f\left(x_{i}\right) r_{i}-\int_{D_{r_{i}}} f\right)\right|=\left|\sum_{D_{r_{i}} \in A}\left(f\left(x_{i}\right) r_{i}-\int_{D_{r_{i}}} f\right)\right| \\
+\left|\sum_{D_{r_{i}} \in B}\left(f\left(x_{i}\right) r_{i}-\int_{D_{r_{i}}} f\right)\right| \leq 2 \varepsilon
\end{aligned}
$$

provando (ii).

Aplicando a desigualdade ||$x|-| y|| \leq|x-y|$ a (ii), obtemos (iii).

Corolário 2.28. Se $f$ é integrável em $D$ e $\int_{D_{1}} f=0$, qualquer que seja o disco $D_{1} \subset D$. Então $|f|$ é integrável em $D$ e $\int_{D}|f|=0$

Prova. Dado $\varepsilon>0$ seja $\delta$ o gauge em $D$ satisfazendo a definição de integral. Então, qualquer que seja $\mathcal{D}=\left\{\left(x_{1}, D_{r_{1}}\right), \ldots,\left(x_{n}, D_{r_{n}}\right)\right\}<<\delta$ temos, por hipótese e por (i) do Lema de Henstock,

$$
\left|f\left(x_{i}\right) \cdot r_{i}-\int_{D_{r_{i}}} f\right|=\left|f\left(x_{i}\right)\right| \cdot r_{i}
$$

para cada $1 \leq i \leq n$.

Segue-se por (ii) do mesmo Lema que

$$
2 \varepsilon \geq \sum_{i=1}^{n}\left|f\left(x_{i}\right) r_{i}-\int_{D_{r_{i}}} f\right|=\sum_{i=1}^{n}\left(\left|f\left(x_{i}\right)\right| \cdot r_{i}\right),
$$

provando que $|f|$ é integrável e sua integral é nula.

\subsection{As funções absolutamente integráveis}

Definição 2.29. Uma função $f: D \rightarrow \mathbb{R}$ é chamada de absolutamente integrável se $f$ e $|f|$ são integráveis. 
O próximo teorema estabelece condições para que uma função integrável seja absolutamente integrável. Nesse teorema denotaremos por $\Psi$ o conjunto de todas as coleções finitas de discos contidos em $D$, dois a dois disjuntos. É imediato que qualquer divisão de $D$ é um elemento de $\Psi$.

Teorema 2.30. Scjam $f$ e $g$ funções integráveis em $D$ tais que $|f(x)| \leq g(x)$, para todo $x \in D$. Então,

(i) $\left|\int_{D} f\right| \leq \int_{D} g$;

(ii) $\lambda=\sup \left\{\sum_{i=1}^{n}\left|\int_{D_{i}} f\right|:\left\{D_{1}, \ldots, D_{n}\right\} \in \Psi\right\} \leq \int_{D} g$,

(iii) $|f|$ é integrável em D e $\int_{D}|f|=\lambda \leq \int_{D} g$.

Prova. (i) Dado $\varepsilon>0$ seja $\delta$ um gauge que satisfaz a definição de integral para as funções $f$ e $g$, isto é, para toda divisão marcada $\delta$-fina $\mathcal{D}$,

$$
\left|S(f, \mathcal{D})-\int_{D} f\right|<\frac{\varepsilon}{2} \quad \text { e } \quad\left|S(g, \mathcal{D})-\int_{D} g\right|<\frac{\varepsilon}{2}
$$

Observando que para toda divisão marcada $\mathcal{D}$ é válido $S(|f|, \mathcal{D}) \leq S(g, \mathcal{D})$, temos

$$
\begin{aligned}
\left|\int_{D} f\right| & <\frac{\varepsilon}{2}+|S(f, \mathcal{D})| \leq \frac{\varepsilon}{2}+|S(|f|, \mathcal{D})| \\
& \leq \frac{\varepsilon}{2}+|S(g, \mathcal{D})| \leq \frac{\varepsilon}{2}+\left|-\int_{D} g+S(g, \mathcal{D})\right|+\left|\int_{D} g\right| \\
& <\varepsilon+\left|\int_{D} g\right|
\end{aligned}
$$

Da arbitrariedade de $\varepsilon>0$ segue-se que $\left|\int_{D} f\right| \leq \int_{D} g$, encerrando a prova de (i)

(ii) De (2.16) e (i) temos $\left|\int_{E} f\right| \leq \int_{E} g$, para todo disco $E \subset D$. Logo, se $D_{1}, \ldots, D_{n}$ são discos contidos em $D$, dois a dois disjuntos, então, por (2.21), vem

$$
\sum_{i=1}^{n}\left|\int_{D_{i}} f\right| \leq \sum_{i=1}^{n} \int_{D_{i}} g=\int_{D} g
$$


Pela definição de supremo, segue-se que

$$
\lambda=\sup \left\{\sum_{i=1}^{n}\left|\int_{D_{i}} f\right|:\left\{D_{1}, \ldots, D_{n}\right\} \in \Psi\right\} \leq \int_{D} g,
$$

encerrando a prova de (ii).

(iii) Inicialmente verifiquemos que para cada $\varepsilon>0$ existe uma divisão $\mathcal{D}$ de $D$ satisfazendo

$$
\sum_{i=1}^{n}\left|\int_{D_{i}} f\right|>\lambda-\frac{\varepsilon}{5}
$$

De fato, pela definição de supremo existe uma divisão $\left\{D_{1}, \ldots, D_{m}\right\} \in \Psi$ tal que (1) é válida. Se $\bigcup_{i=1}^{m} D_{r_{i}} \neq D$ então, por (1.23), existem $D_{m+1}, \ldots, D_{n}$ tais que $\bigcup_{i=1}^{n} D_{r_{i}}=D$ e $\lambda \geq \sum_{i=1}^{n}\left|\int_{D_{i}} f\right| \geq \sum_{i=1}^{m}\left|\int_{D_{i}} f\right|>\lambda-\frac{\varepsilon}{5}$, verificando (1).

Seja $\stackrel{i=1}{\mathcal{D}}=\left\{D_{1}, \ldots, D_{m}\right\}$ a tal divisão e seja $\delta_{1}$ um gauge em $D$ satisfazendo a definição da integral para $\frac{\varepsilon}{5}$.

Para cada $x \in D$ existe um único $1 \leq i \leq n$ tal que $x \in D_{i}$. Assim, recordando (1.5) e (1.6.ii) definamos o gauge $\delta$ em $D$ por

$$
\delta(x)=\left\{\begin{array}{lll}
\delta_{1}(x) & \text { se } & \delta_{1}(x) \subset D_{i} \\
D_{i} & \text { se } & D_{i} \subset \delta_{1}(x)
\end{array}\right.
$$

Qualquer que seja $\mathcal{D}_{1}=\left\{\left(x_{1}, D_{r_{1}}\right), \ldots,\left(x_{m}, D_{r_{m}}\right)\right\}<<\delta$ temos por (1.5.i) que, para cada $1 \leq k \leq m$, existe um único índice $i$ verificando $D_{r_{k}} \subset D_{i}$. Pela linearidade da integral,

$$
\int_{D_{i}} f=\sum_{D_{r_{k}} \subset D_{i}} \int_{D_{r_{k}}} f
$$

$\mathrm{e}$

$$
\left|\int_{D_{i}} f\right| \leq \sum_{D_{r_{k}} \subset D_{i}}\left|\int_{D_{r_{k}}} f\right|
$$

De $\left\{D_{r_{1}}, \ldots, D_{r_{m}}\right\} \in \Psi$ segue

$$
\lambda-\frac{\bar{\varepsilon}}{5}<\sum_{i=1}^{n}\left|\int_{D_{i}} f\right| \leq \sum_{i=1}^{n} \sum_{D_{r_{k}} \subset D_{i}}\left|\int_{D_{r_{k}}} f\right|=\sum_{k=1}^{m}\left|\int_{D_{r_{k}}} f\right| \leq \lambda
$$


Consideremos as divisões $\mathcal{D}_{1}=\left\{\left(x_{1}, D_{r_{1}}\right), \ldots,\left(x_{n}, D_{r_{n}}\right)\right\}<<\delta$, $\mathcal{D}_{2}=\left\{\left(y_{1}, D_{s_{1}}\right), \ldots,\left(y_{m}, D_{s_{m}}\right)\right\}<<\delta$ e provemos que $S\left(|f|, \mathcal{D}_{1}\right)$ e $S\left(|f|, \mathcal{D}_{2}\right)$ verificam a condição de Cauchy.

De $\mathcal{D}_{1}$ e $\mathcal{D}_{2}$ verificarem (3) vem

$$
\left|\sum_{i=1}^{n}\right| \int_{D_{r_{i}}} f\left|-\sum_{j=1}^{m}\right| \int_{D_{s_{j}}} f||<\frac{\varepsilon}{5}
$$

Pelo Lema de Henstock,

$$
\sum_{i=1}^{n}|| f\left(x_{i}\right)\left|\cdot r_{i}-\right| \int_{D_{r_{i}}} f||<\frac{2 \varepsilon}{5}
$$

e

$$
\sum_{j=1}^{m}|| f\left(y_{j}\right)\left|\cdot s_{j}-\right| \int_{D_{s_{j}}} f||<\frac{2 \varepsilon}{5}
$$

Portanto, usando (4),(5) e (6), vem

$$
\begin{aligned}
& \left|S\left(|f|, D_{1}\right)-S\left(|f|, D_{2}\right)\right|=\left|\sum_{i=1}^{n}\right| f\left(x_{i}\right)\left|\cdot r_{i}-\sum_{j=1}^{m}\right| f\left(y_{j}\right)\left|\cdot s_{j}\right| \\
& \leq\left|\sum_{i=1}^{n}\right| f\left(x_{i}\right)\left|\cdot r_{i}-\sum_{i=1}^{n}\right| \int_{D_{r_{i}}} f\left|+\sum_{j=1}^{m}\right| \int_{D_{s_{j}}} f\left|-\sum_{j=1}^{m}\right| f\left(y_{j}\right)\left|\cdot s_{j}\right| \\
& +\left|\sum_{i=1}^{n}\right| \int_{D_{r_{i}}} f\left|-\sum_{j=1}^{m}\right| \int_{D_{s_{j}}} f|| \\
& \leq \sum_{i=1}^{n}|| f\left(x_{i}\right)\left|\cdot r_{i}-\right| \int_{D_{r_{i}}} f||+\sum_{j=1}^{m}|| f\left(y_{j}\right)\left|\cdot s_{j}-\right| \int_{D_{s_{j}}} f||+\frac{\varepsilon}{5} \\
& <\frac{2 \varepsilon}{5}+\frac{2 \varepsilon}{5}+\frac{\varepsilon}{5}=\varepsilon
\end{aligned}
$$

mostrando que $|f|$ é integrável.

Dadas as funções $f, g: D \rightarrow \mathbb{R}$, é usual definirmos as funções $f \vee g$, $f \wedge g: D \rightarrow \mathbb{R}$ por

$$
(f \vee g)(x)=\sup \{f(x), g(x)\}
$$

e

$$
(f \wedge g)(x)=\inf \{f(x), g(x)\}
$$

para todo $x \in D$. 
Corolário 2.31. Sejam $f_{1}, f_{2}, g, h: D \rightarrow \mathbb{R}$ funções integráveis. Se, para todo $x \in \mathcal{D}$, tem-se $g(x) \leq f_{i}(x) \leq h(x), i=1,2$, então as funções $\left|f_{1}-f_{2}\right|$, $f_{1} \vee f_{2}$ e $f_{1} \wedge f_{2}$ são integráveis.

Prova. Para todo $x \in D$, tem-se $\left|f_{1}(x)-f_{2}(x)\right| \leq h(x)-g(x)$. Como $g$ e $h$ são integráveis, segue-se por (2.12.i) que $h-g \geq 0$ é integrável. Pelo teorema anterior, $\left|f_{1}-f_{2}\right|$ é integrável.

Como

$$
f_{1} \vee f_{2}=\frac{1}{2}\left(f_{1}+f_{2}+\left|f_{1}-f_{2}\right|\right)
$$

$$
f_{1} \wedge f_{2}=\frac{1}{2}\left(f_{1}+f_{2}-\left|f_{1}-f_{2}\right|\right),
$$

então, por (2.12.i), $f_{1} \vee f_{2}$ e $f_{1} \wedge f_{2}$ são integráveis.

Corolário 2.32. Sejam $\left(f_{n}\right)_{n \geq 1}$ uma seqüência de funções integráveis em $D$. Se existem $g, h: D \rightarrow \mathbb{R}$ funções integráveis tais que, para todo $x \in D$ e todo $n \geq 1, g(x) \leq f_{n}(x) \leq h(x)$, então $f_{i} \vee \ldots \vee f_{k}$ e $f_{i} \wedge \ldots \wedge f_{k}$ são integráveis em $D$, para todo $1 \leq i<k$.

Prova. Segue do corolário anterior. 


\section{Capítulo 3}

\section{Teoremas de Convergência}

\subsection{Conjunto Nulo}

Definição 3.1. Diz-se que um subconjunto $N$ de $D$ é um conjunto nulo se, para todo $\varepsilon>0$, existe uma seqüência de discos $\left(D_{r_{i}}\right)_{i \geq 1}$, com $D_{r_{i}} \subset D$ para todo $i \geq 1$, satisfazendo:
(i) $\quad N \subset \bigcup_{i=1}^{\infty} D_{r_{i}}$;
(ii) $\sum_{i=1}^{\infty} r_{i}<\varepsilon$.

Observação 3.2. Considerando (1.5) podemos sempre assumir que os discos da seqüência são dois a dois disjuntos.

Exemplo 3.3. O conjunto dos números naturais é um conjunto nulo em $\mathbb{Z}_{p}$.

Em $\mathbb{Z}_{p}$, todo número natural é da forma $\sum_{i=0}^{n} a_{i} p^{i}$, com $a_{n} \neq 0$. Assim, para cada $n \geq 0$, a quantidade de números naturais tais que $a_{n} \neq 0$ é dada 
por

$$
p^{n}(p-1)
$$

Isso posto, dado $\varepsilon>0$, seja $m \in \mathbb{N}$ satisfazendo a condição

$$
\frac{1}{p^{m}} \cdot \frac{p}{p-1}<\varepsilon
$$

A cada número $\sum_{i=0}^{n} a_{i} p^{i}$, (é claro $a_{n} \neq 0$ ) associemos um disco contendo esse número e de raio $\frac{1}{p^{m+2 n+1}}$.

Como para cada $n$ a quantidade de discos com tais raios é dado por (1), a soma dos raios desses discos é

$$
\frac{p^{n}(p-1)}{p^{m+2 n+1}} \leq \frac{1}{p^{m+n}}
$$

para cada $n$.

Logo, usando (2), a soma dos raios de todos os discos será limitada por

$$
\sum_{n=0}^{\infty} \frac{1}{p^{m+n}}=\frac{1}{p^{m}} \sum_{n=1}^{\infty} \frac{1}{p^{n}}=\frac{1}{p^{m}} \cdot \frac{p}{p-1}<\varepsilon
$$

satisfazendo (3.1.ii).

Pela construção dos discos é claro que (3.1.i) está verificada. Assim $\mathbb{N}$ é um conjunto nulo e, relembremos, denso em $\mathbb{Z}_{p}$.

Definição 3.4. Diz-se que uma propriedade P é válida quase sempre para os elementos de um conjunto, e escreve-se $P$ q.s., se o conjunto dos pontos para os quais ela não é válida é um conjunto nulo.

Teorema 3.5. Se $f=g$ q.s. e $g \in \mathbb{K} \mathbb{H}(D)$ então $f \in \mathbb{K H}(D)$ e $\int_{D} f=\int_{D} g$. 
Prova. Inicialmente suponhamos que $g=0 \mathrm{em} D$ e seja $N \subset D$ tal que $f(x) \neq 0$ para todo $x \in N$. Dado $\varepsilon>0$ seja $E_{s_{1}}, \ldots, E_{s_{m}}, \ldots$, uma seqüência de discos contidos em $D$, dois a dois disjuntos, satisfazendo (i) e (ii) de (3.1) para esse $\varepsilon$

Para cada $j \geq 1$ e cada $x \in N \cap E_{s_{j}}, 1 \leq j \leq m$, seja

$$
m(x)=\frac{s_{j}}{|f(x)|+1}
$$

Definamos, em $D$, o gauge $\delta$ por

$$
\delta(x)=\left\{\begin{array}{lll}
B_{1}(x) & , \text { se } & x \notin N ; \\
\left.B_{m(x}\right)(x) & \text {, se } & x \in N .
\end{array}\right.
$$

Então, dada $\mathcal{D}=\left\{\left(x_{1}, D_{r_{1}}\right), \ldots,\left(x_{n}, D_{r_{n}}\right)\right\}<<\delta$ e observando que $r_{j}<m\left(x_{j}\right)$, se $x_{j} \in N$, temos

$$
\begin{array}{r}
\left|\sum_{i=1}^{n} f\left(x_{i}\right) r_{i}\right|=\left|\sum_{x_{i} \notin N} f\left(x_{i}\right) r_{i}+\sum_{x_{j} \in N} f\left(x_{j}\right) r_{j}\right|=\left|\sum_{x \in N} f\left(x_{j}\right) r_{j}\right| \\
\leq \sum_{x_{j} \in N}\left(\left|f\left(x_{j}\right)\right| \cdot \frac{s_{j}}{\left|f\left(x_{j}\right)\right|+1}\right)<\sum_{x_{j} \in N} s_{j}<\sum_{j=1}^{\infty} s_{j}<\varepsilon
\end{array}
$$

provando o teorema para esse caso.

Agora, se $g \neq 0$ consideramos a função $h=f-g$. Temos $h=0$ q.s. e, como $f=g+(f-g)$, a conclusão segue usando (2.12.i).

Corolário 3.6. Se $N$ é um conjunto nulo, então $\chi_{N}$ é integrável em $D$ e $\int_{D} \chi_{N}=0$.

Prova. Imediata.

\subsection{Teoremas de Convergência}

A seguir apresentamos os teoremas de convergência. Esses teoremas fornecem condições suficientes para a permuta das operações de limite e integral. 
Teorema 3.7. Seja $\left(f_{n}\right)_{n \geq 1}$ uma seqüência de funções integráveis definidas em $D$ satisfazendo:

(i) $f_{n}(x) \rightarrow f(x) \quad$ q.s.;

(ii) dado $\varepsilon>0$, existe um gauge $\delta$ em $D$ tal que a cada $\mathcal{D}<<\delta$ está associado um número natural $n_{\mathcal{D}}$ tal que, para todo $n \geq n_{\mathcal{D}}$ a desigualdade

$$
\left|S\left(f_{n}, \mathcal{D}\right)-\int_{D} f_{n}\right|<\varepsilon
$$

está satisfeita.

Nessas condições, $f$ é integrável em $D$ e $\int_{D} f=\lim _{n \rightarrow \infty} \int_{D} f_{n}$

Prova. Suponhamos a condição (i) satisfeita para todo $x \in D$. Provemos que $\lim _{n \rightarrow \infty} \int_{D} f_{n}$ existe e é finito.

Dado $\varepsilon>0$, sejam $\delta$ um gauge e $\mathcal{E}=\left\{\left(x_{i}, D_{r_{i}}\right): i=1, \ldots, s\right\}<<\delta$, fixada, e $n_{\mathcal{E}}$ satisfazendo (ii).

Dados $m, n \geq n_{\mathcal{E}}$ temos

$$
\begin{aligned}
& \left|\int_{D} f_{m}-\int_{D} f_{n}\right| \leq\left|\int_{D} f_{m}-\sum_{i=1}^{s} f_{m}\left(x_{i}\right) \cdot r_{i}\right|+\left|\sum_{i=1}^{s} f_{m}\left(x_{i}\right) \cdot r_{i}-\sum_{i=1}^{s} f_{n}\left(x_{i}\right) \cdot r_{i}\right| \\
& +\left|\sum_{i=1}^{s} f_{n}\left(x_{i}\right) \cdot r_{i}-\int_{D} f_{n}\right| \\
& <2 \cdot \varepsilon+\left|\sum_{i=1}^{s}\left[\left(f_{m}\left(x_{i}\right)-f_{n}\left(x_{i}\right)\right) \cdot r_{i}\right]\right|
\end{aligned}
$$

Por (i), para cada $1 \leq i \leq s$ existe um $n\left(x_{i}\right)$ tal que

$$
\left|f_{m}\left(x_{i}\right)-f_{n}\left(x_{i}\right)\right|<\frac{\varepsilon}{s \cdot r_{i}}
$$

quaisquer que sejam $m, n \geq n\left(x_{i}\right)$.

Tomando-se $m, n \geq \max \left\{n_{x_{1}}, \ldots, n_{x_{s}}, n_{\mathcal{E}}\right\}$ segue de (1) e (2) que,

$$
\left|\int_{D} f_{m}-\int_{D} f_{n}\right|<2 \cdot \varepsilon+\left|\sum_{i=1}^{s}\left[\left(f_{m}\left(x_{i}\right)-f_{n}\left(x_{i}\right)\right) \cdot r_{i}\right]\right|
$$




$$
\leq 2 \cdot \varepsilon+\left|\sum_{i=1}^{s}\left[\frac{\varepsilon}{s \cdot r_{i}} \cdot r_{i}\right]\right|<2 \cdot \varepsilon+\varepsilon=3 \cdot \varepsilon
$$

Isso mostra que a seqüência $\left(\int_{D} f_{n}\right)_{n \geq 1}$ é de Cauchy em $\mathbb{R}$, logo convergente. Seja $I=\lim _{n \rightarrow \infty} \int_{D} f_{n}$.

A seguir mostraremos que $f$ é integrável'.

Dado $\varepsilon>0$ seja $n_{0}$ tal que é válida a desigualdade

$$
\left|\int_{D} f_{n}-I\right|<\frac{\varepsilon}{3}
$$

para todo $n \geq n_{0}$.

Seja $\delta$ um gauge satisfazendo (ii) para $\frac{\varepsilon}{3}$.

Qualquer que seja $\mathcal{D}=\left\{\left(z_{i}, D_{r_{i}}\right): i=1, \ldots, k\right\}<<\delta$, determinamos $n_{1}$ de modo que

$$
\left|f\left(z_{i}\right)-f_{n}\left(z_{i}\right)\right|<\frac{\varepsilon}{3 \cdot 2^{i} \cdot r_{i}}
$$

esteja satisfeita para todo $i=1, \ldots k$, e $n \geq n_{1}$.

Finalmente, escolhendo $n=\max \left\{n_{\mathcal{D}}, n_{0}, n_{1}\right\}$ vem

$$
\begin{aligned}
& |S(f, \mathcal{D})-I| \leq\left|S(f, \mathcal{D})-S\left(f_{n}, \mathcal{D}\right)\right|+\left|S\left(f_{n}, \mathcal{D}\right)-\int_{D} f_{n}\right|+\left|\int_{D} f_{n}-I\right| \\
& \leq \sum_{i=1}^{k}\left|f\left(z_{i}\right)-f_{n}\left(z_{i}\right)\right| \cdot r_{i}+\left|S\left(f_{n}, \mathcal{D}\right)-\int_{D} f_{n}\right|+\left|\int_{D} f_{n}-I\right| \\
& <\sum_{i=1}^{k} \frac{\varepsilon}{2^{i} \cdot 3}+\frac{\varepsilon}{3}+\frac{\varepsilon}{3}<\varepsilon
\end{aligned}
$$

Isso encerra a prova para $\lim _{n \rightarrow \infty} \int_{D} f_{n}(x)=\int_{D} f(x)$, para todo $x \in D$.

Assumamos que (i) é válida e denotemos por $N$ o conjunto dos $x \in D$ tais que $\lim _{n \rightarrow \infty} f_{n}(x) \neq f(x)$.

Definamos

$$
g_{n}(x)= \begin{cases}f_{n}(x) & , \text { se } x \in D-N \\ 0 & , \text { se } x \in N\end{cases}
$$

$\mathrm{e}$

$$
g(x)= \begin{cases}f(x) & , \text { se } x \in D-N \\ 0 & , \text { se } x \in N\end{cases}
$$


É imediato que

$$
g_{n}(x)=f_{n}(x) \text { q.s. } \quad \text { e } \quad g(x)=f(x) \text { q.s. }
$$

e $g_{n}(x) \rightarrow g(x)$, para todo $x \in D$.

Mostremos que $g_{n}(x)$ verifica (ii). Dado $\varepsilon>0$, seja $\delta$ o gauge tal que cada $\mathcal{D}=\left\{\left(x_{i}, D_{r_{i}}\right): i=1, \ldots, m\right\}<<\delta$ e $n_{\mathcal{D}}$ verificando (*) para as funções $f_{u}$. Tomemos $k \geq n_{\mathcal{D}}$. Lembrando que $\int_{D} f_{n}=\int_{D} g_{n}$, para cada $n \geq 1$, e o lema de Henstock, vem:

$$
\begin{aligned}
& \left|S\left(g_{n}, \mathcal{D}\right)-\int_{D} g_{n}\right|=\left|\sum_{i=1}^{m}\left(g_{n}\left(x_{i}\right) r_{i}-\int_{D_{r_{i}}} g_{n}\right)\right| \\
& \leq\left|\sum_{x_{i} \in D-N}\left(g_{n}\left(x_{i}\right) r_{i}-\int_{D_{r_{i}}} g_{n}\right)\right|+\left|\sum_{x_{i} \in N}\left(g_{n}\left(x_{i}\right) r_{i}-\int_{D_{r_{i}}} g_{n}\right)\right| \\
& \leq\left|\sum_{x_{i} \in D-N}\left(f_{n}\left(x_{i}\right) r_{i}-\int_{D_{r_{i}}} f_{n}\right)\right| \\
& \leq\left|\sum_{x_{i} \in D-N}\left(f_{n}\left(x_{i}\right) r_{i}-\int_{D_{r_{i}}} f_{n}\right)\right|+\left|\sum_{x_{i} \in N}\left(f_{n}\left(x_{i}\right) r_{i}-\int_{D_{r_{i}}} f_{n}\right)\right| \\
& <2 \varepsilon
\end{aligned}
$$

mostrando que (ii) é válida para a seqüência $\left(g_{n}\right)_{n>1}$. Portanto, pelas considerações primeiras, $g$ é integrável e $\int_{D} g=\lim _{k \rightarrow \infty} \int_{D} g_{n}$. Usando (3), segue-se que $f$ é integrável e $\int_{D} f=\int_{D} g=\lim _{k \rightarrow \infty} \int_{D} g_{n}=\lim _{k \rightarrow \infty} \int_{D} f_{n}$.

Definição 3.8. Seja $\left(f_{n}\right)_{n \geq 1}$ uma seqüência de funções integráveis em $D$. Diz-se $\left(f_{n}\right)_{n \geq 1}$ é uniformemente integrável em $D$ se, para cada $\varepsilon>0$ existe um gauge $\delta$ tal que, para cada $\mathcal{D}<<\delta$, tem-se $\left|S\left(f_{n}, \mathcal{D}\right)-\int_{D} f_{n}\right|<\varepsilon$ para todo $n \geq 1$.

Corolário 3.9. Se $\left(f_{n}\right)_{n \geq 1}$ é uniformemente integrável em $D$ e $\lim _{n \rightarrow \infty} f_{n}=f$, então $f$ é integrável em $D$ e $\int_{D} f=\lim _{n \rightarrow \infty} \int_{D} f_{n}$.

Prova. Toma-se $n_{\mathcal{D}}=1$, para toda $\mathcal{D}<<\delta$ c aplica-se $(3.7)$. 
Teorema 3.10. Scja $\left(f_{n}\right)_{n \geq 1}$ uma seqüência de funções integráveis em $D$ que convergem uniformemente para $f$. Se a seqüencia $\left(\int_{D} f_{n}\right)_{n \geq 1}$ converge, então $f$ é integrável em $D$ e $\int_{D} f=\lim _{n \rightarrow \infty} \int_{D} f_{n}$.

Prova. Seja $\varepsilon>0$, arbitrário. A hipótese da convergência uniforme e o Critério de Cauchy implicam na existência de um índice $n_{0} \geq 1$ tal que

$$
\left|f_{m}(x)-f_{n}(x)\right|<\frac{\varepsilon}{3 \cdot r}
$$

quaisquer que sejam $m, n \geq n_{0}$ e $x \in D$.

Da convergência da seqüencia das integrais existe $n_{1} \geq 1$ tal que, quaisquer que sejam $m, n \geq n_{1}$, tem-se

$$
\left|\int_{D} f_{n}-\int_{D} f_{m}\right|<\frac{\varepsilon}{3}
$$

Se $N=\max \left\{n_{0}, n_{1}\right\}$. Então existe um gauge $\delta$ tal que

$$
\left|S\left(f_{N}, \mathcal{D}\right)-\int_{D} f_{N}\right|<\frac{\varepsilon}{3}
$$

qualquer que seja $\mathcal{D}<<\delta$

Para toda $\mathcal{D}=\left\{\left(x_{1}, D_{r_{1}}\right), \ldots,\left(x_{n}, D_{r_{n}}\right)\right\}<<\delta$ façamos $n_{\mathcal{D}}=N$. Então, para todo $n \geq n_{\mathcal{D}}$ tem-se observando (1),(2) e (3),

$$
\begin{aligned}
&\left|S\left(f_{n}, \mathcal{D}\right)-\int_{D} f_{n}\right| \leq \mid S\left(f_{n}, \mathcal{D}\right)-S\left(f_{N}, \mathcal{D}|+| S\left(f_{N}, D\right)-\right. \int_{D} f_{N} \mid \\
&+\left|\int_{D} f_{N}-\int_{D} f_{n}\right| \\
& \leq \sum_{i=1}^{n}\left[\left|f_{n}\left(x_{i}\right)-f_{N}\left(x_{i}\right)\right| \cdot r_{i}\right]+\frac{\varepsilon}{3}+\frac{\varepsilon}{3}<\frac{\varepsilon}{3 r} \cdot \sum_{i=1}^{n} r_{i}+\frac{2 \varepsilon}{3}=\frac{\varepsilon}{3}+\frac{2 \varepsilon}{3}=\varepsilon
\end{aligned}
$$

Estando satisfeitas as hipóteses de (3.7), a conclusão segue-se.

Teorema 3.11. (Teorema da Convergência Monótona) Seja $\left(f_{n}\right)_{n \geq 1}$ uma seqüência de funções integráveis em $D$ satisfazendo as seguintes condições: 
(i) $\quad f_{n}(x) \rightarrow f(x)$, para quase todo $x \in D$;

(ii) $f_{n}(x) \leq f_{n+1}(x)$, para quase todo $x \in D$ e $n \geq 1$.

Então $f$ é integrável em $D$ se e somente se $\lim _{n \rightarrow \infty} \int_{D} f_{n}<\infty$.

Nessas condições $\int_{D} f=\lim _{n \rightarrow \infty} \int_{D} f_{n}$.

Prova. Seja $N$ o conjuntos dos $x \in D$ tais que $f_{n}(x) \nrightarrow f(x)$ ou existe $n \geq 1$ tal que $f_{n}(x) \not \leq f_{n+1}(x)$. Por hipótese esse conjunto é nulo. Definamos

$$
g_{n}(x)=\left\{\begin{array}{lll}
f_{n}(x) & , \text { se } & x \in D-N \\
0 & \text {, se } & x \in N
\end{array}\right.
$$

$$
g_{(}(x)=\left\{\begin{array}{lll}
f(x) & , \text { se } & x \in D-N \\
0 & , \text { se } & x \in N .
\end{array}\right.
$$

Dessa definição, segue-se que as hipótese do teorema, aplicadas a $g$ e $g_{n}$, são válidas para todo $x \in D$.

$(\Rightarrow)$ Suponhamos $f$ e $f_{n}$ integrável em $D$. Por (3.5), $g$ e $g_{n}$ são integráveis em $D$, para todo $n \geq 1$. Lembrando (2.13) vem, para todo $n \geq 1$,

$$
\int_{D} f_{n}=\int_{D} g_{n} \leq \int_{D} g=\int_{D} f<\infty .
$$

e,

$$
\int_{D} f_{n}=\int_{D} g_{n} \leq \int_{D} g_{n+1}=\int_{D} f_{n+1} .
$$

Essas desigualdades mostram que a seqüência $\left(\int_{D} f_{n}\right)_{n \geq 1}$ é monótona crescente e limitada. Portanto existe $\lim _{n \rightarrow \infty} \int_{D} f_{n} \leq \int_{D} f<\infty$.

$(\Leftarrow)$ Temos $\lim _{n \rightarrow \infty} \int_{D} g_{n}=\lim _{n \rightarrow \infty} \int_{D}^{n \rightarrow \infty} f_{n}<\infty$.

Seja $\varepsilon>0$ arbitrário.

Da convergência da seqüência $\left(\int_{D} g_{n}\right)_{n>1}$ segue-se, pelo Critério de Cauchy, que existe $n_{0} \geq 1$ tal que, quaisquer que sejam $m, n \geq n_{0}$,

$$
\left|\int_{D} g_{n}-\int_{D} g_{m}\right|<\frac{\varepsilon}{3}
$$

De $g_{n}$ integrável, segue-se que existe um gauge $\delta_{n}$ tal que, pelo Lema de Henstock, se $\left\{\left(x, E_{s}\right)\right\}<<<\delta_{n}$ então

$$
\left|g_{n}(x) \cdot s-\int_{E_{s}} g_{n}\right|<\frac{\varepsilon}{3 \cdot 2^{n}}
$$


para cada $n \geq 1$,

De (ii), para cada $x \in D$, existe um $n(x) \geq n_{0}$, tal que

$$
\left|f_{m}(x)-f_{n}(x)\right|<\frac{\varepsilon}{3 \cdot r} .
$$

sempre que $m, n \geq n(x)$.

Definamos o gauge $\delta(x)=\delta_{n(x)}(x)$, para todo $x \in D$.

Assim, dada $\mathcal{D}=\left\{\left(x_{1}, D_{r_{1}}\right), \ldots,\left(x_{k}, D_{r_{k}}\right)\right\}<\delta$ tomamos $n_{\mathcal{D}}=\max \left\{n\left(x_{1}\right), \ldots, n\left(x_{k}\right)\right\}$. Portanto, para todo $n \geq n_{\mathcal{D}}$ temos, usando (1), (2), (3) e (2.20)

$$
\begin{aligned}
& \left|S\left(g_{n}, \mathcal{D}\right)-\int_{D} g_{n}\right|=\left|\sum_{i=1}^{k} g_{n}\left(x_{i}\right) \cdot r_{i}-\int_{D} g_{n}\right|=\left|\sum_{i=1}^{k} g_{n}\left(x_{i}\right) \cdot r_{i}-\sum_{i=1}^{k} \int_{D_{r_{i}}} g_{n}\right| \\
& \leq \sum_{i=1}^{k}\left[\left|g_{n}\left(x_{i}\right)-g_{n\left(x_{i}\right)\left(x_{i}\right)}\right| \cdot r_{i}\right]+\left|\sum_{i=1}^{k}\left(g_{n\left(x_{i}\right)}\left(x_{i}\right) \cdot r_{i}-\int_{D_{r_{i}}} g_{n\left(x_{i}\right)}\right)\right| \\
& +\left|\sum_{i=1}^{k}\left(\int_{D_{r_{i}}} g_{n\left(x_{i}\right)}-\int_{D_{r_{i}}} g_{n}\right)\right| \\
& \leq \frac{\varepsilon}{3 \cdot r} \sum_{i=1}^{k} r_{i}+\frac{\varepsilon}{3} \sum_{i=1}^{k} \frac{1}{2^{n\left(x_{i}\right)}}+\left|\sum_{i=1}^{k}\left(\int_{D_{r_{i}}} g_{n_{0}}-\int_{D_{r_{i}}} g_{n}\right)\right| \\
& <\frac{\varepsilon}{3}+\frac{\varepsilon}{3}+\left|\int_{D} g_{n_{0}}-\int_{D} g_{n}\right|<\varepsilon
\end{aligned}
$$

Estão, dessa forma, satisfeitas as condições (i) e (ii) de (3.7). Segue-se que $g$ é integrável e $\int_{D} g=\lim _{n \rightarrow \infty} \int_{D} g_{n}$.

Finalmente, de (3.5), vem:

$$
\int_{D} f=\int_{D} g=\lim _{n \rightarrow \infty} \int_{D} g_{n}=\lim _{n \rightarrow \infty} \int_{D} f_{n}
$$

encerrando a prova do teorema.

Exemplo 3.12. Seja $\left(D_{r_{i}}\right)_{i \geq 1}$ uma seqüência de discos, dois a dois disjuntos, tais que $D_{r_{i}} \subset D$, para todo $i \geq 1$. Mostremos que $\chi_{i=1}^{\infty} D_{r_{i}}$ é integrável em $D$ e $\int_{D} \chi_{i=1}^{\infty} D_{r_{i}}=\sum_{i=1}^{\infty} r_{i}$ 
Iniciemos observando que, de acordo com (1.23), existem, para cada $n \geq 1$, $\operatorname{discos} F_{s_{1}}^{n}, \ldots, F_{s_{m}}^{n}$, dois a dois disjuntos satisfazendo

$$
D=\bigcup_{i=1}^{n} D_{r_{i}} \bigcup\left(\bigcup_{j=1}^{m} F_{s_{j}}^{n}\right)
$$

e, por (1.29),

$$
r=\sum_{i=1}^{n} r_{i}+\sum_{j=1}^{m} s_{j} .
$$

Segue-se, dessa igualdade, que $\sum_{i=1}^{n} r_{i}<r$, para todo $n \geq 1$. Daí

$$
\sum_{i=1}^{\infty} r_{i}=\lim _{n \rightarrow \infty} \sum_{i=1}^{n} r_{i} \leq r
$$

Agora, pelo exemplo (2.10), $\chi_{D_{r_{i}}}$ é integrável em $D$, para cada $i \geq 1$.

Definamos, para cada $x \in D$,

$$
\begin{aligned}
& f_{1}(x)=\chi_{D_{r_{1}}}(x) \\
& f_{n}(x)=\chi_{\bigcup_{i=1}^{n} D_{r_{i}}}(x) \quad \text {, se } n>1 .
\end{aligned}
$$

Observando que $\chi_{\bigcup_{i=1}^{n} D_{r_{i}}}(x)=\sum_{i=1}^{n} \chi_{D_{r_{i}}}(x)$, para todo $x \in D$ e todo $n>1$, vem, por (2.12.i), que

$$
f_{n} \text { é integrável em } D \text {, para todo } n \geq 1
$$

Ainda da definição de $f_{n}$ seguem:

$$
f_{n}(x) \leq f_{n+1}(x), \text { para todo } x \in D
$$

e

$$
f_{n}(x)=\chi_{\bigcup_{i=1}^{n} D_{r_{i}}} \rightarrow f(x)=\chi \chi_{i=1}^{\infty} D_{r_{i}}
$$


Com (2),(3) e (4) estão satisfeitas as hipóteses do TCM. Finalmente, lembrando (1),

$$
\begin{aligned}
\lim _{n \rightarrow \infty} \int_{D} f_{n}=\lim _{n \rightarrow \infty} \int_{D} \chi_{\bigcup_{i=1}^{n} D_{r_{i}}}(x) & =\lim _{n \rightarrow \infty} \int_{D}\left(\sum_{i=1}^{n} \chi_{D_{r_{i}}}(x)\right) \\
= & \left.\lim _{n \rightarrow \infty} \sum_{i=1}^{n} \int_{D} \chi_{D_{r_{i}}}(x)\right)=\lim _{n \rightarrow \infty} \sum_{i=1}^{n} r_{i}=\sum_{i=1}^{\infty} r_{i} \leq r .
\end{aligned}
$$

Segue-se, pelo TCM que $\chi_{\bigcup_{i=1}^{\infty} D_{r_{i}}}$ é integrável em $D$ e $\int_{D} \chi_{\bigcup_{n=1}^{\infty} D_{r_{i}}}=\sum_{i=1}^{\infty} r_{i}$, como queríamos verificar.

Exemplo 3.13. Seja $f: \mathbb{Z}_{p} \rightarrow \mathbb{R}$ definida por

$$
f(x)=\sum_{i=0}^{\infty} \frac{a_{i}}{p^{2 i}}, \quad \text { se } x=\sum_{i=0}^{\infty} a_{i} p^{i} .
$$

Tomando $|x-y|_{p}=p^{-n}$, vem:

$$
\begin{aligned}
|f(x)-f(y)| & =\sum_{i=n}^{\infty} \frac{a_{i}}{p^{2 i}} \leq(p-1) \sum_{i=n}^{\infty} \frac{1}{p^{2 i}} \\
& =\frac{p-1}{p^{2 n}} \cdot \sum_{i=0}^{\infty} \frac{1}{p^{i}}=\frac{p-1}{p^{2 n}} \cdot \frac{p}{p-1} \\
& =\frac{1}{p^{n-1}} \cdot p^{-n}=\frac{1}{p^{n-1}} \cdot|x-y|_{p}
\end{aligned}
$$

mostrando que $f$ é uniformemente contínua em $D$.

Segue-se por (2.24) que $f$ é integrável em $\mathbb{Z}_{p}$.

Determinemos $\int_{D} f$.

Consideremos a seqüência $\left(f_{n}(x)\right)_{n \geq 1}$ definida por

$$
f_{n}(x)=\left(a_{0}+\frac{a_{1}}{p^{2}}+\ldots+\frac{a_{n-1}}{p^{2(n-1)}}\right) \cdot \chi_{B_{p-n}\left(a_{0}+a_{1} p+\ldots+a_{n-1} p^{n-1}\right)}
$$

se $x=a_{0}+a_{1} p+\ldots+a_{n-1} p^{n-1}+a_{n} p^{n}+\ldots$

Para cada $n \geq 1$, temos 


$$
\begin{aligned}
& \int_{\mathbb{Z}_{p}} f_{n}(x) \\
& =\sum_{i_{1}, \ldots, i_{n}=0}^{p \ldots, p}\left(a_{i_{1}}+\frac{a_{i_{2}}}{p^{2}}+\ldots+\frac{a_{i_{n}}}{p^{2(n-1)}}\right) \cdot \int_{\mathbb{Z}_{p}} \chi_{B_{p^{-n}}\left(a_{i_{1}}+\ldots+a_{i_{n}} p^{n-1}\right)} \\
& =\sum_{i_{1}, \ldots, p}\left(a_{i_{1}}+\frac{a_{i_{2}}}{p^{2}}+\ldots+\frac{a_{i_{n}}}{p^{2(n-1)}}\right) \cdot p^{-n} \\
& =p^{n-1} \cdot \frac{(p-1) p}{2} \cdot p^{-n} \cdot\left(1+\ldots+\frac{1}{p^{2(n-1)}}\right) \\
& =\frac{p-1}{2} \cdot \frac{1-\frac{1}{p^{2(n-1)}}}{1-\frac{1}{p^{2}}} \\
& =\frac{p-1}{2} \cdot \frac{p^{2} \cdot\left(p^{2(n-1)}-1\right)}{p^{2(n-1)} \cdot\left(p^{2}-1\right)}
\end{aligned}
$$

É imediato que a seqüência é monótona crescente e

$$
\lim _{n \rightarrow \infty} \int_{\mathbb{Z}_{p}} f_{n}=\frac{p-1}{2} \cdot \frac{p^{2}}{p^{2}-1}=\frac{p}{2(p+1)}
$$

Estando satisfeitas as hipóteses do TCM segue-se que $\int_{\check{z}_{p}} f=\frac{p}{2(p+1)}$.

Corolário 3.14. Seja $\left(f_{n}\right)_{n \geq 1}$ uma seqüência de funções integráveis em $D$ satisfazendo as seguintes condições:

(i) $f_{n}(x) \rightarrow f(x)$, para quase todo $x \in D$;

(ii) $f_{n}(x) \geq f_{n+1}(x)$, para quase todo $x \in D$ e $n \geq 1$.

Então, $f$ é integrável em $D$ se e somente se $\lim _{n \rightarrow \infty} \int_{D} f_{n}>-\infty$.

Definimos $g(x)=f_{1}(x)-f(x)$ e $g_{n}(x)=f_{1}(x)-f_{n}(x)$, para todo $n \geq 1$. Segue-se que $\int_{D} g_{n}$ existe para todo n e $g$ é integrável se e somente se $f$ for integrável. Por outro lado a seqüência de funções $\left(g_{n}\right)_{n \geq 1}$ satisfaz as hipóteses de (3.11). Logo

$$
\int_{D} g=\lim _{n \rightarrow \infty} g_{n} \Leftrightarrow \lim _{n \rightarrow \infty} \int_{D} g_{n}<\infty
$$

Segue-se

$$
\begin{aligned}
f \quad \text { é integrável } \Leftrightarrow g \quad \text { é integrável } & \Leftrightarrow \lim _{n \rightarrow \infty} \int_{D} g_{n}<\infty \\
& \Leftrightarrow \lim _{n \rightarrow \infty} \int_{D} f_{n}=\int_{D} f_{1}-\lim _{n \rightarrow \infty} \int_{D} g_{n}<\infty
\end{aligned}
$$


Lema 3.15. (Lema de Fatou) Seja $\left(f_{n}\right)_{n \geq 1}$ uma seqüência de funções integráveis em $D$ e sejam $g_{1}, g_{2}$ funções integráveis $\mathrm{cm} D$ tais que $g_{1}(x) \leq f_{n}(x) \leq g_{2}(x)$, para quase todo $x \in D$ e todo $n \geq 1$. Então $\lim \inf f_{n}$ é integrável em $D$ e $\int_{D} \liminf f_{n} \leq \liminf \int_{D} f_{n}$.

Prova. Por (3.5) não há perda de generalidade admitirmos $g_{1}(x) \leq f_{n}(x) \leq g_{2}(x)$, para todo $x \in D$ e todo $n \geq 1$. Assim, para cada par $(n, m)$ de números naturais, com $1 \leq n \leq m$ e todo $x \in D$ definamos

$$
f_{n}^{m}(x)= \begin{cases}f_{n}(x) & , \text { se } m=n \\ \left(f_{n} \wedge \ldots \wedge f_{m}\right)(x) & \text {, se } m>n\end{cases}
$$

Quaisquer que sejam $m \geq n \geq 1$, usando (2.32), temos

$$
f_{n}^{m} \text { é integrável em } D \text {, }
$$

e, de $f_{n}^{m}(x) \geq g_{1}(x)$, utilizando (2.13), vem

$$
\int_{D} f_{n}^{m} \geq \int_{D} g_{1}
$$

Fixados $n$ e $x$, a seqüência $\left(f_{n}^{m}(x)\right)_{m>n}$ é monótona decrescente e limitada inferiormente por $g_{1}(x)$. Portanto está bem definida a função

$$
h_{n}(x)=\inf \left\{f_{n}^{m}(x): m \geq n\right\}
$$

Além disso, essa seqüência satisfaz as hipóteses de (3.14). Assim, a função $h_{n}(x)$ é integrável em $D$ e satisfaz as condições

$$
g_{1}(x) \leq h_{n}(x) \leq g_{2}(x), \text { para todo } n \geq 1 .
$$

De $\int_{D} h_{n} \leq \int_{D} f_{n}^{m}$, para todo $m \geq n$ segue-se

$$
\int_{D} h_{n} \leq \inf _{m \geq n} \int_{D} f_{m}
$$

de $\inf \left\{f_{n}^{m}: m \geq n\right\} \leq \inf \left\{f_{n+1}^{m}: m \geq n+1\right\}$ vem que a seqüência $\left(h_{n}(x)\right)_{n \geq 1}$ é monótona crescente e limitada superiormente por $g_{2}(x)$, convergindo para $\sup \left\{h_{n}(x): n \geq 1\right\}=\liminf f_{n}$. 
Estão, dessa forma, satisfeitas as hipóteses do TCM, com $\lim _{n \rightarrow \infty} \int_{D} h_{n} \leq$ $\int_{D} g_{2}<\infty$. Segue-se, pelo mesmo tcorema, que $\lim \inf f_{n}$ é integrável e, observando (4),

$$
\int_{D} \liminf f_{n}=\lim _{n \rightarrow \infty} \int_{D} h_{n} \leq \lim _{n \rightarrow \infty} \inf _{m \geq n} \int_{D} \inf f_{n}=\liminf \int_{D} f_{n}
$$

completando a prova do teorema.

Corolário 3.16. Seja $\left(f_{n}\right)_{n \geq 1}$ uma seqüência de funções integráveis em $D$ e sejam $g_{1}, g_{2}$ funções integráveis em $D$ tais que $g_{1}(x) \leq f_{n}(x) \leq g_{2}(x)$, para quase todo $x \in D$ e todo $n \geq 1$. Então $\limsup f_{n}$ é integrável em $D$ e $\int_{D} \lim \sup f_{n} \geq \lim \sup \int_{D} f_{n}$.

Prova. Assumamos, sem perda de generalidade, $g_{1}(x) \leq f_{n}(x) \leq g_{2}(x)$, para todo $x \in D$. Para todo $x \in D$, a seqüência de funções $h_{n}(x)=-f_{n}(x)$, com $n \geq 1$, e as funções $l_{1}(x)=-g_{1}(x), l_{2}(x)=-g_{2}(x)$ satisfazem as hipóteses do Lema de Fatou. Portanto

$$
-\int_{D} \liminf h_{n} \leq \liminf \int_{D} h_{n}
$$

Lembrando que $\lim \inf h_{n}=-\limsup f_{n}$ e que $\liminf \int_{D} f_{n}=-\limsup \int_{D} f_{n}$, segue-se, de (1)

ou

$$
\int_{D}-\limsup f_{n} \leq-\limsup \int_{D} f_{n}
$$

$$
\int_{D} \limsup f_{n} \geq \limsup \int_{D} f_{n}
$$

provando o corolário.

Teorema 3.17. (Teorema da Convergência Dominada) Seja $\left(f_{n}\right)_{n \geq 1}$ uma seqüência de funções integráveis em $D$ e sejam $g_{1}, g_{2}$ funções integráveis em $D$ tais que $g_{1}(x) \leq f_{n}(x) \leq g_{2}(x)$, para quase todo $x \in D$ e todo $n \geq 1$. Se $\lim _{n \rightarrow \infty} f_{n}(x)=f(x)$, cntão $f$ é integrável e $\int_{D} f=\lim _{n \rightarrow \infty} \int_{D} f_{n}$. 
Prova. Estão satisfeitas as hipóteses do Lema de Fatou e do seu corolário. Como $f=\liminf f_{n}=\limsup f_{n}$, segue-se que $f$ é integrável em $D$. Usando o Lema e o seu corolário, temos

$$
\int_{D} f=\int_{D} \liminf f_{n} \leq \liminf \int_{D} f_{n} \leq \limsup \int_{D} f_{n} \leq \int_{D} \limsup f_{n}=\int_{D} f
$$

Isso mostra que

$$
\liminf \int_{D} f_{n}=\limsup \int_{D} f_{n}=\int_{D} f
$$

Da primcira igualdade temos

$$
\lim _{n \rightarrow \infty} \int_{D} f_{n}
$$

existe; e a segunda que esse limite é igual a $\int_{D} f$ provando o teorema. 


\section{Capítulo 4}

\section{Integração em conjuntos}

\subsection{Funções mensuráveis}

Uma função $e: D \rightarrow \mathbb{R}$ é chamada de função escada se ela é dada por uma combinação linear finita de funções características de discos, isto é, para todo $x \in D$,

$$
e(x)=\sum_{i=1}^{n} a_{i} \chi_{D_{r_{i}}}(x)
$$

$\operatorname{com} a_{i} \in \mathbb{R}$.

É imediato que toda função escada é absolutamente integrável.

Definição 4.1. Uma função $f: D \rightarrow \mathbb{R}$ é chamada de mensurável se existe uma seqüência de funções escada que converge para $f$ q.s.

Decorre dessa definição que as funções características de discos assim como as funções escadas são mensuráveis.

Teorema 4.2. Toda função contínua é mensurável.

Prova. Seja $r=p^{k}$, com $k \in \mathbb{Z}$, o raio do disco $D$. Por (1.29) existem, para cada $n \geq 1, D_{1}^{n}, \ldots, D_{p^{n}}^{n}$, discos de raio $p^{k-n}$, dois a dois disjuntos, 
satisfazendo $D=\bigcup_{i=1}^{p^{n}} D_{i}^{n}$.

Designando por $m_{i}^{n}=\inf \left\{f(x): x \in D_{i}^{n}\right\}$, definimos a função escada

$$
f(x)=\sum_{i=1}^{p^{n}} m_{i}^{n} \chi_{D_{i}^{n}}(x),
$$

para cada $x \in D$.

Provemos que $\lim _{n \rightarrow \infty} e_{n}(x)=f(x)$, para todo $x \in D$.

Fixado $x$ existe, para cada $n \geq 1$, um índice $1 \leq i(n) \leq p^{n}$ tal que

$$
x \in D_{i(n)}^{n} \cap D_{i(n+1)}^{n+1}
$$

Como $p^{k-(n+1)}<p^{k-n}$ segue-se, por (1.5.i), que $D_{i(n+1)}^{n+1} \subset D_{i(n)}^{n}$. Logo $m_{i(n)}^{n} \leq m_{i(n+1)}^{(n+1)} \leq f(x)$ e a seqüência $\left(m_{i(n)}^{n}\right)_{n \geq 1}$ é monótona crescente e limitada, portanto convergente.

A continuidade de $f$ e a compacidade de $D$ implicam que $f$ é uniformemente contínua. Assim, dado $\varepsilon>0$ existe $n_{0} \in \mathbb{Z}$ tal que, para todo $y \in$ $D_{i\left(n_{0}\right)}^{n_{0}}$ temos $|f(x)-f(y)|<\varepsilon$. A compacidade de $D_{i\left(n_{0}\right)}^{n_{0}}$ acarreta a existência de $y \in D_{i\left(n_{0}\right)}^{n_{0}}$ satisfazendo $f(y)=m_{i\left(n_{0}\right)}^{n_{0}}$. Portanto, $\left|f(x)-m_{i\left(n_{0}\right)}^{n_{0}}\right|<\varepsilon$ e o crescimento monótono de $m_{i(n)}^{n}$ permite-nos concluir que, para todo $n \geq n_{0}$, temos $\left|f(x)-m_{i(n)}^{n}\right|<\varepsilon$, mostrando que $e_{n}(x) \rightarrow f(x)$ quando $n \rightarrow \infty$, completando a prova do teorema.

Teorema 4.3. Se $f$ é mensurável e existem $g, h: D \rightarrow \mathbb{R}$ satisfazendo $g(x) \leq f(x) \leq h(x)$ q.s., com $g, h \in \mathbb{K} \mathbb{H}(D)$, então $f \in \mathbb{K} \mathbb{H}(D)$.

Prova. . Assumamos, sem perda de generalidade, $g(x) \leq f(x) \leq h(x)$, para todo $x \in D$.

A mensurabilidade de $f$ acarreta a existência de uma seqüência $\left(e_{n}\right)_{n \geq 1}$ de funções escada satisfazendo $\lim _{n \rightarrow \infty} e_{n}=f$.

Definindo-se $h_{n}(x)=e_{n} \wedge h$, temos, por (2.31), que $h_{n}$ é integrável em $D$ e satisfaz $g(x) \leq h_{n}(x) \leq h(x)$, para cada $n \geq 1$ e $x \in D$.

Pela sua definição, temos $h_{n} \rightarrow f$, quando $n \rightarrow \infty$. Estando satisfeitas as hipóteses do TCD, segue-se que $f$ é integrável em $D$. 
O exemplo a seguir exibe uma função mensurável, não limitada superiormente, logo não verifica a hipótese $g(x) \leq f(x) \leq h(x)$, e que não é integrável. Isso mostra que essa hipótese não pode ser eliminada no teorema acima.

Exemplo 4.4. Em $\mathbb{Z}_{3}$ consideremos a função $f$ definida por

$$
f(x)=\left\{\begin{array}{lll}
3 & \text {, se } \quad x=2+\sum_{j=1}^{\infty} b_{j} \cdot 3^{j} \\
\frac{3^{n}}{2^{n-1}} \sum_{k=2^{n-1}-1}^{2^{n}-2} \frac{1}{2 k+1} & \text {, se } x=\sum_{i=0}^{n-2} a_{i} \cdot 3^{i}+2 \cdot 3^{n-1}+\sum_{j=n}^{\infty} b_{j} \cdot 3^{j} \\
0 & \text { fora }
\end{array}\right.
$$

no qual os $a_{i} \in\{0,1\}$, para todo $i$, e $b_{j} \in\{0,1,2\}$, para todo $j$. Em todo o restante do texto, para não sermos prolixos, $a_{i}$ e $b_{j}$ tomarão sempre os valores aqui expressos.

É fácil notarmos que $f$ é constante em todo disco de raio $3^{-n}$ contendo os pontos em que o algarismo 2 surge, pela primeira vez, como coeficiente do termo $3^{n-1}$.

Mostraremos que essa função é mensurável, porém não é integrável.

Definamos a seqüência de funções $\left(g_{n}\right)_{n \geq 1}$ em $D$ da seguinte forma:

$$
g_{1}(x)=\left\{\begin{array}{lll}
3 & , \text { se } & x=2+\sum_{j=1}^{\infty} b_{j} \cdot 3^{j} \\
0 & \text { fora }
\end{array}\right.
$$

e, para todo $n>1$,

$$
g_{n}(x)= \begin{cases}\frac{3^{n}}{2^{n-1}} \cdot \sum_{k=2^{n-1}}^{2^{n-2}} \frac{1}{2 k+1} & , \text { se } \quad x=\sum_{i=0}^{n-2} a_{i} \cdot 3^{i}+2 \cdot 3^{n-1}+\sum_{j=n}^{\infty} b_{j} \cdot 3^{j} \\ 0 & \text { fora }\end{cases}
$$


É imediato que essas funções são funções escadas. A partir dessas funções construiremos uma seqüência de funções escadas que convergem para $f$.

Para todo $x \in \mathbb{Z}_{3}$, definamos

$$
e_{n}(x)=\left\{\begin{array}{lll}
g_{1}(x) & \text { se } & n=1 \\
\sum_{k=1}^{n} g_{k}(x) & \text { se } & n>1
\end{array}\right.
$$

Para todo $n, e_{n}$ é uma função escada e $\lim _{n \rightarrow \infty} e_{n}(x)=f(x)$ para todo $x \in \mathbb{Z}_{3}$. Isso mostra que $f$ é mensurável.

Por outro lado $e_{n}(x) \leq e_{n+1}(x)$, para todo $x \in \mathbb{Z}_{3}$, mostrando que a seqüência $\left(\left(e_{n}(x)\right)_{n \geq 1}\right.$ é monótona crescente. Além disso,

$$
\int_{\mathbb{Z}_{3}} e_{n}=\sum_{k=0}^{2^{n}-2} \frac{1}{2 k+1}<\infty
$$

satisfazendo as hipóteses do TC.M. Entretanto, de

$$
\lim _{n \rightarrow \infty} \int_{\mathbb{Z}_{3}} e_{n}=\sum_{k=0}^{\infty} \frac{1}{2 k+1}=\infty
$$

segue-se, pelo TCM, que $f$ não é integrável, concluindo o exemplo.

\subsection{Conjuntos mensuráveis}

Nesta seção introduziremos uma $\sigma$-álgebra em $D$ e definiremos o conceito de conjunto mensurável. Para tanto, denotaremos

$$
\mathcal{A}=\left\{E \subset D: \quad \chi_{E} \in \mathbb{K} \mathbb{H}(D)\right\}
$$

Teorema 4.5. $\mathcal{A}$ é uma $\sigma$-álgebra $\mathrm{cm} D$. 
Prova. . É imediato que $\emptyset, D \in \mathcal{A}$. Agora, se $A \in \mathcal{A}$ então $\chi_{A} \in \mathbb{K} \mathbb{H}(D)$. Logo, por (2.12.i), $\chi_{\bar{A}}=1-\chi_{A} \in \mathbb{K} \mathbb{H}(D)$, mostrando que $\tilde{A} \in \mathcal{A}$.

Dados $A_{1}, \ldots, A_{n} \in \mathcal{A}$ segue-se $\chi_{A_{1}}, \ldots, \chi_{A_{n}} \in \mathbb{K} \mathbb{H}(D)$. De $0 \leq \chi_{A_{i}}(x) \leq$ 1 , para todo $x \in D$, segue-se, por (2.32),

$$
\chi_{n=1}^{n} A_{n}=\chi_{A_{1}} \vee \ldots \vee \chi_{A_{n}} \in \mathbb{K} \mathbb{H}(D) .
$$

Isso mostra que $\chi_{\bigcup_{n=1}^{n} A_{n}} \in \mathcal{A}$.

Seja $\left(A_{n}\right)_{n \geq 1}$ uma seqüência de elementos de $\mathcal{A}$.

Definamos

$$
f_{n}(x)= \begin{cases}\chi_{A_{1}} & \text {, se } n=1 \\ \chi_{i=1}^{n} A_{i} & \text {, se } n \geq 1 .\end{cases}
$$

Temos, então, que $\left(f_{n}\right)_{n \geq 1}$ é uma seqüência monótona crescente de funções integráveis verificando $f_{n}(x) \leq \chi_{D}(x)$, para todo $x \in D$ e, por (2.13), $\int_{D} f_{n} \leq \int_{D} \chi_{D}=r$. Segue-se, pelo TCM,

$$
\int_{D} \chi \bigcup_{n=1}^{\infty} A_{n}=\int_{D} \lim _{n \rightarrow \infty} \chi \bigcup_{k=1}^{n} A_{k}=\lim _{n \rightarrow \infty} \int_{D} \chi_{\bigcup_{k=1}^{n} A_{k}}=\lim _{n \rightarrow \infty} \int_{D} f_{n} \leq r
$$

mostrando que $\bigcup_{n=1}^{\infty} A_{n} \in \mathcal{A}$ e provando que $\mathcal{A}$ é uma $\sigma$-álgebra. D.

O próximo teorema mostra que a $\sigma$-álgebra $\mathcal{A}$ contém os borelianos de

Teorema 4.6. A $\sigma$-álgebra $\mathcal{A}$ contém os borelianos $\mathcal{B}$ de $D$.

Prova. . É suficiente verificarmos que $\mathcal{A}$ contém os abertos de $D$. Por (1.30), para todo subconjunto aberto $A$ de $D$ existe uma seqüência $\left(D_{i}\right)_{i \geq 1}$ de discos contidos em $D$, dois a dois disjuntos, satisfazendo $A=\bigcup_{n=1}^{\infty} D_{n}$. De $D_{n} \in \mathcal{A}$ segue-se, pelo teorema anterior, que $A \in \mathcal{A}$.

Diz-se que um subconjunto $A$ de $D$ é mensurável se $A \in \mathcal{A}$. Com essa definição, introduziremos uma medida sobre $\mathcal{A}$ fazendo

$$
\mu(A)=\int_{D} \chi_{A}
$$

para todo $A \in \mathcal{A}$. 
Teorema 4.7. $\mu$ é uma medida completa sobre $D$.

Prova. É claro que $\mu$ é $\sigma$-aditiva, pois se $A_{1}, \ldots, A_{n}, \ldots$ uma seqüência de elementos de $\mathcal{A}$, dois a dois disjuntos,temos

$$
\begin{aligned}
\mu\left(\bigcup_{n=1}^{\infty} A_{n}\right)= & \int_{D} \chi_{\bigcup_{n=1}^{\infty} A_{n}}=\int_{D} \lim _{m \rightarrow \infty} \chi_{\bigcup_{n=1}^{m} A_{n}}=\lim _{m \rightarrow \infty} \int_{D} \chi_{\bigcup_{n=1}^{m} A_{n}} \\
& =\lim _{m \rightarrow \infty} \int_{D} \sum_{n=1}^{m} \chi_{A_{n}}=\lim _{m \rightarrow \infty} \sum_{n=1}^{m} \int_{D} \chi_{A_{n}}=\lim _{m \rightarrow \infty} \sum_{n=1}^{m} \mu_{A_{n}}=\sum_{n=1}^{\infty} \mu_{A_{n}}
\end{aligned}
$$

Seja $A \in \mathcal{A}, \operatorname{com} \mu(A)=0$, e seja $B$ um subconjunto de $A$. Provemos que $B$ é mensurável e $\mu(B)=0$. É suficiente verificarmos que $\chi_{B}$ é integrável em D.

De $\mu(A)=0$ temos $\int_{D} \chi_{A}=0$. Logo, dado $\varepsilon>0$ existe $\delta$ um gauge que satisfaz a definição de integral para a função $\chi_{A}$. Então, para toda divisão $\mathcal{D}=\left\{\left(x_{1}, D_{r_{1}}\right), \ldots,\left(\xi_{n}, D_{r_{n}}\right)\right\}<<\delta$, vem

$$
\left|\sum_{i=1}^{n} \chi_{B}\left(\xi_{i}\right) r_{i}\right| \leq\left|\sum_{i=1}^{n} \chi_{A}\left(\xi_{i}\right) r_{i}\right|<\varepsilon .
$$

mostrando que $\chi_{B}$ é integrável e a sua integral é nula.

Portanto todo subconjunto de um conjunto de medida nula é mensurável e a medida é completa.

Proposição 4.8. Todo subconjunto fechado de um espaço métrico compacto é compacto. Reciprocamente, um subconjunto compacto de qualquer espaço métrico é fechado

Prova. Vide ([9])p.211, Proposição 1.

Segue-se dessa proposição o corolário.

Corolário 4.9. Seja $A \subset D$. $A$ é fechado se e somente se $A$ é compacto.

Proposição 4.10. Scja $X$ um espaço de Hausdorff no qual cada subconjunto abcrto $A$ é um $F_{\sigma}$ e seja $\nu$ uma medida Borel finita sobre $X$. Então, para 
cada $A \in \mathcal{B}(X)$, tem-se:

(i) $\quad \nu(A)=\inf \{\nu(U): A \subset U$ e $U$ é aberto $\}$;

(ii) $\nu(A)=\sup \{\nu(U): F \subset A$ e $\mathrm{F}$ é fechado $\}$;

Prova. . Ver [7], p.207, Lema 7.2.4.

Teorema 4.11. A medida $\mu$ é regular sobre os borelianos de $D$.

Prova. . Por (1.30) todo aberto $A \subset D$ é um $F_{\sigma}$. Estão, pois, satisfeitas as hipóteses da proposição (4.10). E como, pela proposição (4.8), todo fechado em D é compacto, segue-se que estão satisfeitas as condições da definição de medida regular. Portanto $\mu$ é uma medida regular sobre os borelianos de D.

Teorema 4.12. Sejam $A \in \mathcal{A}$ então $\chi_{z+A}$ é integrável e $\int_{z+D} \chi_{z+A}=\int_{D} \chi_{A}$.

Prova. . De $\chi_{A}$ integrável segue-se que, dado $\varepsilon>0$ existe um gauge $\delta_{1}$ satisfazendo a definição de integral de $\chi_{A}$ para esse $\varepsilon$.

Definamos em $z+D$ o gauge $\delta$ por $\delta(z+x)=z+\delta_{1}(x)$, para todo $x \in D$. Segue-se, para todo $x \in D$,

$$
D_{s} \subset \delta_{1}(x) \quad \text { se e somente se } \quad z+D_{s} \subset z+\delta_{1}(x)=\delta(z+x)
$$

Para toda divisão $\delta$-fina de $z+D$,

$$
\left\{\left(z+x_{1}, z+D_{r_{1}}\right), \ldots,\left(z+x_{n}, z+D_{r_{n}}\right)\right\},
$$

usando (1) e lembrando o teorema (1.31), vem:

$$
\left|\sum_{i=1}^{n} \chi_{z+A}\left(z+x_{i}\right) r_{i}-\int_{D} \chi_{A}\right|=\left|\sum_{i=1}^{n} \chi_{A}\left(x_{i}\right) r_{i}-\int_{D} \chi_{A}\right|<\varepsilon
$$

provando que $\chi_{z+A}$ é integrável e que $\int_{z+D} \chi_{z+A}=\int_{D} \chi_{A}$.

Os teoremas (4.11) e (4.12) mostram que a medida $\mu$ definida em $\mathcal{A}$ é a medida de Haar.

Em (4.6) mostramos todo subconjunto boreliano de um disco é mensurável. Uma questão natural que surge é sabermos se todos os subconjuntos de um disco são mensuráveis. O exemplo a seguir responde essa questão e a resposta, já prevista, é negativa. 
Exemplo 4.13. Existe $A \subset \mathbb{Z}_{p}$ não mensurável.

Prova. Definamos em $\mathbb{Z}_{p}$ a relação

$$
x \cong y \text { se e somente se } x-y \in \mathbb{Z}
$$

É imediato que $\cong \dot{e}$ uma relação de equivalência em $\mathbb{Z}_{p}$.

Para cada $x \in \mathbb{Z}_{p}$ seja

$$
[x]=\left\{y \in \mathbb{Z}_{p}: y \cong x\right\}
$$

Como $\mathbb{Z}$ é denso em $\mathbb{Z}_{p}$ então $\mathbb{Z}+x$ também é denso em $\mathbb{Z}_{p}$. Seja $E \subset \mathbb{Z}_{p}$ o conjunto obtido escolhendo-se um elemento de cada classe de equivalência e seja

$$
E_{n}=E+n, \text { para cada } n \geq 1
$$

Temos

$$
E_{n} \cap E_{m}=\emptyset, \text { se } n \neq m
$$

De fato, se $x+n \in E_{n} \cap E_{m}$ então, existe $y \in E$ tal que $x+n=y+m$. Logo,

$$
x+n=y+m \Rightarrow x-y=m-n \in \mathbb{Z} \Rightarrow x \in[y],
$$

contrariando a escolha dos elementos em $E$

É claro que $\bigcup_{n=1}^{\infty} E_{n} \subset \mathbb{Z}_{p}$.

Provemos a inclusão oposta.

Se $x \in \mathbb{Z}_{p}$ então existe $e \in E$ tal que

$$
x \cong e \Rightarrow x-e \in \mathbb{Z} \Rightarrow x=e+k, \text { para algum } k \in \mathbb{Z}
$$


Portanto $x \in E_{k}$ e $\mathbb{Z}_{p} \subset \bigcup_{n=1}^{\infty} E_{n}$. Logo,

$$
\bigcup_{n=1}^{\infty} E_{n}=\mathbb{Z}_{p}
$$

Provemos que $E$ não é mensurável.

Suponhamos, por absurdo, que $E$ seja mensurável. Então $\chi_{E}$ é integrável.

Decorre disso que $\chi_{E_{n}}$ é integrável e

$$
\int_{\mathbb{Z}_{p}} \chi_{E_{n}}=\int_{\mathbb{Z}_{p}} E
$$

Definamos, para todo $x \in \mathbb{Z}_{p}$,

$$
f_{m}(x)= \begin{cases}\chi_{E_{1}}(x) & , \text { se } m=1 \\ \chi_{\bigcup_{n=1}^{m} E_{n}}(x) & , \text { se } m>1 .\end{cases}
$$

Por (2.12.i) $f_{m}$ é integrável e

$$
\int_{\mathbb{Z}_{p}} f_{m}=\int_{\mathbb{Z}_{p}} \chi_{\bigcup_{n=1}^{m} E_{n}}=\int_{\mathbb{Z}_{p}} \sum_{n=1}^{m} \chi_{E_{n}}=\sum_{n=1}^{m} \int_{\mathbb{Z}_{p}} \chi_{E_{n}}=m \cdot \int_{\mathbb{Z}_{p}} \chi_{E}
$$

Por outro lado, quaisquer que sejam $x \in \mathbb{Z}_{p}$ e $m \geq 1$ temos

$$
f_{m}(x)=\chi_{\bigcup_{n=1}^{m} E_{n}}(x) \leq \chi_{\mathbb{Z}_{p}}(x)
$$

Destas duas últimas relações segue-se que a desigualdade

$$
m \int_{\mathbb{Z}_{p}} \chi_{E} \leq \int_{\mathbb{Z}_{p}} \chi_{\mathbb{Z}_{p}}=1
$$

é válida para todo $m \geq 1$. Portanto $\int_{Z_{p}} \chi_{E}=0$. Isso acarreta que $\chi_{E}=0$ q.s. e, portanto $E$ é um conjunto nulo (veja (4.23)). Este resultado, (1) e (2) implicam que $\mathbb{Z}_{p}$ também é um conjunto nulo, o que é uma contradição. Portanto $E$ não é mensurável. $\diamond$ 


\subsection{Integrabilidade e mensurabilidade}

O objetivo desta seção é provar que toda função integrável é mensurável. Ela é iniciada com as notações que serão utilizadas no decorrer da mesma.

Notações 4.14. Seja $f$ integrável em $D$. Designaremos:

(a) por $\Gamma$ a família dos pares ordenados $(x, E)$, na qual $E$ é um disco contido em $D$ e $x \in E$;

(b) para cada $\varepsilon>0$,

$$
\Gamma_{\frac{1}{\varepsilon}}=\left\{\left(x, E_{s}\right) \in \Gamma:\left|\int_{E_{s}} f-f(x) \cdot s\right| \geq \varepsilon \cdot s\right\}
$$

Em particular, para todo $k \geq 1$,

$$
\Gamma_{k}=\left\{\left(x, E_{s}\right) \in \Gamma:\left|\int_{E_{s}} f-f(x) \cdot s\right| \geq \frac{s}{k}\right\}
$$

(c) para cada par $k, n \geq 1$,

$$
\Gamma_{k n}=\left\{\left(x, E_{s}\right) \in \Gamma_{k}: E_{s} \subset B\left(x, p^{-n}\right)\right\}
$$

(d) e, para cada par $k, n \geq 1$,

$$
A_{k n}=\left\{x \in D: \text { existe um disco } E_{s} \text { tal que }\left(x, E_{s}\right) \in \Gamma_{k n}\right\}
$$

(e) por $A_{k}=\bigcap_{n=1}^{\infty} A_{k n}$, para todo $k \geq 1$.

Decorre de (4.14.(b)) a propriedade:

$$
\text { se } k \leq m \quad \text { então } \quad \Gamma_{k} \subset \Gamma_{m}
$$


Lema 4.15. Seja $f$ integrável em $D$. Então, quaisquer que sejam $\eta>0$ e $\varepsilon>0$, existe um gauge $\delta$ tal que, para toda $\mathcal{E}=\left\{\left(x, E_{s}\right):\left(x, E_{s}\right) \in \Gamma_{\frac{1}{\eta}}\right\}<<<$ $\delta$ tem-se $\sum_{\left(x . E_{s}\right) \in \mathcal{E}} s<\varepsilon$.

Prova. Seja $\eta>0$ arbitrário. Da integrabilidade de $f$, dado $\varepsilon>0$ existe um gauge $\delta$ satisfazendo a definiçãa de integral para $\frac{\varepsilon \cdot \eta}{2}$. Pelo Lema de Henstock, se $\mathcal{E}=\left\{\left(x, E_{s}\right):\left(x, E_{s}\right) \in \Gamma_{\frac{1}{n}}\right\}<<<\delta$, então

$$
\sum_{\left(x, E_{s}\right) \in \mathcal{E}} s=\frac{1}{\eta} \sum_{\left(x, E_{s}\right) \in \mathcal{E}} \eta \cdot s \leq \frac{1}{\eta} \cdot \sum_{\left(x, E_{s}\right) \in \mathcal{E}}\left|\int_{E_{s}} f-f(x) \cdot s\right|<\frac{1}{\eta} \cdot 2 \cdot \frac{\varepsilon \cdot \eta}{2}=\varepsilon,
$$

e a prova do lema está completa.

Lema 4.16. Sejam $k \geq 1$ e $f$ integrável em $D$. Então, dado $\varepsilon>0$ existe um gauge $\delta$ tal que para toda $\mathcal{E}=\left\{\left(x, E_{s}\right): x \in A_{k}\right\}<<<\delta$ tem-se $\sum_{\left(x, E_{s}\right) \in \mathcal{E}} s<\varepsilon$.

Prova. Por (4.15), para cada $n \geq 1$ existe um gauge $\delta_{n}$ tal que

$$
\sum_{\left(x, E_{s}\right) \in \mathcal{E}_{n}} s<\frac{1}{n}
$$

para toda $\mathcal{E}=\left\{\left(x, E_{s}\right):\left(x, E_{s}\right) \in \Gamma_{n}\right\}<<<\delta_{n}$.

Dado $\varepsilon>0$ escolhamos $n(\varepsilon)$ satisfazendo $n(\varepsilon) \geq \max \left\{k, \frac{1}{\varepsilon}\right\}$ e definamos o gauge

$$
\delta(x)=\delta_{n(\varepsilon)}(x), \text { para todo } x \in D
$$

Assim, dada $\mathcal{E}=\left\{\left(x, E_{s}\right): x \in A_{k}\right\}<<<\delta$, segue-se, usando (4.14.1) que $\mathcal{E} \subset \Gamma_{k} \subset \Gamma_{n(\varepsilon)}$ e daí vem $\sum_{\left(r . E_{*}\right) \in \mathcal{E}} s<\frac{1}{n(\varepsilon)}<\varepsilon$, provando o lema.

Teorema 4.17. Se $f$ integrável em $D$ então, $A_{k}$ é um conjunto nulo, para cada $k \geq 1$.

Prova. Fixemos $k \geq 1$. Por (4.16) para todo $\varepsilon>0$ existe um gauge $\delta$ tal que para todo par $\left\{\left(x, E_{s(x)}\right)\right\}<<<\delta$, com $x \in A_{k}$, tem-se $s(x)<\varepsilon$. 
Seja $\Omega=\left\{\left(x, E_{s(x)}\right): x \in A_{k}\right\}$. É claro que $A_{k} \subset \underset{\left(x, E_{s(x)}\right) \in \Omega}{\bigcup} E_{s(x)}$.

Suponhamos determinados $\left(x_{1}, E_{s\left(x_{1}\right)}\right) \ldots,\left(x_{n}, E_{s\left(x_{n}\right)}\right) \operatorname{em} \Omega$.

Se $A_{k} \subset \bigcup_{i=1}^{n} E_{s\left(x_{i}\right)}$ então, como $\left\{\left(x_{i}, E_{s\left(x_{i}\right)}\right): 1 \leq i \leq n\right\}<<<\delta$, segue-se por (4.16) que

$$
\sum_{i=1}^{n} s\left(x_{i}\right)<\varepsilon
$$

e, por (3.1), $A_{k}$ é um conjunto nulo, provando teorema.

Caso contrário, seja

$$
\Omega_{n}=\left\{\left(x, D_{s(x)}\right) \in \Omega: D_{s(x)} \cap \bigcup_{i=1}^{n} E_{s\left(x_{i}\right)}=\emptyset\right\}
$$

$$
0<\omega_{n}=\sup \left\{s(x):\left(x, D_{s(x)}\right) \in \Omega_{n}\right\} \leq r
$$

Escolhamos $\left(x_{n+1}, D_{s\left(x_{n+1}\right)}\right) \in \Omega_{n}$ satisfazendo

$$
s\left(x_{n+1}\right)>\frac{\omega_{n}}{2}
$$

e verifiquemos se $A_{k} \subset \bigcup_{i=1}^{n+1} E_{s\left(x_{i}\right)}$. Se a inclusão for válida o processo se encerra. Se não, continuamos o processo obtendo as seqüências $x_{1}, \ldots, x_{n}, x_{n+1}, \ldots$ e $\omega_{n}, \omega_{n+1}, \ldots$.

Se existir $n_{0}>n$ tal que $A_{k} \subset \bigcup_{i=1}^{n_{0}} E_{s\left(x_{i}\right)}$ então, de (1), $A_{k}$ é um conjunto nulo.

Se não existir $n_{0}$ satisfazendo as condições acima, então construímos uma seqüência de discos, dois a dois disjuntos, $E_{s\left(x_{1}\right)}, \ldots, E_{s\left(x_{n}\right)}, \ldots$, com $x_{i} \in$ $D_{s\left(x_{i}\right)}$. Decorre daí que $\bigcup_{i=1}^{\infty} E_{s\left(x_{i}\right)} \subset D$, e como $\chi_{D_{s\left(x_{i}\right)}}$ existe, para cada $1 \leq$ $i \leq n$, temos, pelo Teorema da Convergência Dominada, que

$$
r \geq \int_{D} \chi_{\bigcup_{i=1}^{\infty} D_{s\left(x_{i}\right)}}=\lim _{m \rightarrow \infty} \sum_{i=1}^{m} \int_{D} \chi_{D_{s\left(x_{i}\right)}}=\sum_{i=1}^{\infty} s\left(x_{i}\right)
$$

mostrando que a série $\sum_{i=1}^{\infty} s\left(x_{i}\right)$ é convergente. logo

$$
\lim _{i \rightarrow \infty} s\left(x_{i}\right)=0
$$


Escolhamos o inteiro $m$ de modo que

$$
\sum_{i=m+1}^{\infty} s\left(x_{i}\right)<\frac{\varepsilon}{2}
$$

e façamos

$$
B=A_{k}-\bigcup_{i=1}^{m} E_{s\left(x_{i}\right)}
$$

Se mostrarmos que $B$ é um conjunto nulo, o teorema está provado. então $A_{k}=B \cup \bigcup_{i=1}^{m} E_{s\left(x_{i}\right)}$ é um conjunto nulo e o teorema está verificado.

Para cada $j>m$ tomemos o disco $F_{r\left(x_{j}\right)}$, com $x_{j} \in F_{r\left(x_{j}\right)}$ e $r\left(x_{j}\right)=2 s\left(x_{j}\right)$. De (4) vem

$$
\sum_{j=m+1}^{\infty} r\left(x_{j}\right)=2 \cdot \sum_{j=m+1}^{\infty} s\left(x_{j}\right)<2 \cdot \frac{\varepsilon}{2}=\varepsilon
$$

e a série $\sum_{j=m+1}^{\infty} r\left(x_{j}\right)$ é convergente.

De $\Omega$ é uma cobertura de $A$ temos que para cada $x \in B$ existe $D_{s(x)}$ tal que $\left(x, D_{s(x)}\right) \in \Omega_{m}$.

Afirmamos que $D_{s(x)} \cap F_{r\left(x_{j}\right)} \neq \emptyset$, para algum $j>m$.

De fato, se tal não ocorresse, teríamos, para todo $j>m, D_{s(x)} \cap F_{r\left(x_{j}\right)}=\emptyset$. Isso implica, usando (2) e (3) que

$$
0<s(x) \leq 2 \cdot \frac{\omega_{j}}{2}<2 \cdot s\left(x_{j+1}\right)=r\left(x_{j+1}\right)
$$

e a série $\sum_{j=m+1}^{\infty} r\left(x_{j}\right)$ é divergente, que é uma contradição.

Seja $N>m$ satisfazendo $D_{s(x)} \cap E_{s\left(x_{N}\right)} \neq \emptyset$ e $D_{s(x)} \cap E_{s\left(x_{N-1}\right)}=\emptyset$.

Novamente, usando (2) e (3), temos

$$
s(x) \leq \omega_{N-1}<r\left(x_{N}\right)
$$

mostrando, por (1.5), que $D_{s(x)} \subset F_{r\left(x_{N}\right)}$. Logo $B \subset \bigcup_{j=1}^{\infty} F_{r\left(x_{j}\right)}$ e, como (5) é válida, segue-se por (3.1), que $B$ é um conjunto nulo, encerrando prova do teorema. 
Corolário 4.18. Para todo $k \geq 1$ seja $A_{k}$ como no teorema anterior. Então $A=\bigcup_{k=1}^{\infty} A_{k}$ é um conjunto nulo.

Prova. Imediata.

Observação 4.19. Seja $f$ é uma função integrável em $D$. Pela definição da integral, para cada $n \geq 1$, existe um gauge $\delta_{n}$ satisfazendo as seguintes condições:

$$
\delta_{n}(x) \subset B_{p^{-n}}(x) \text {, para todo } x \in D
$$

e, qualquer que seja $\mathcal{D}_{n}=\left\{\left(x_{i}, E_{s_{i}}\right): i=1, \ldots, \alpha(n)\right\}<<\delta_{n}$,

$$
\left|S\left(f, \mathcal{D}_{n}\right)-\int_{D} f\right|<\frac{1}{n}
$$

Fixemos, para cada $n \geq 1$,

$$
\mathcal{D}_{n}=\left\{\left(x_{i}, E_{s_{i}}\right): i=1, \ldots, \alpha(n)\right\}<<\delta_{n}
$$

e definamos

$$
\phi_{n}(x)=\sum_{i=1}^{\alpha(n)} \frac{\int_{E_{s_{i}}} f}{s_{i}} \cdot \chi_{E_{s_{i}}}(x)
$$

É imediato que a $\phi_{n}(x)$ é uma função escada e, para cada $x \in D$, existe um único $1 \leq i \leq \alpha(n)$ tal que $x \in E_{s_{i}},\left(x_{i}, E_{s_{i}}\right) \in \mathcal{D}_{n} \mathrm{e}$

$$
\phi_{n}(x)=\frac{\int_{E_{s_{i}}} f}{s_{i}}
$$

Consideremos a seqüência $\left(\phi_{n}(x)\right)_{n \geq 1}$ e designemos por

$$
N=\left\{x \in D: \lim _{n \rightarrow \infty} \phi_{n}(x) \neq f(x),\right\}
$$

no qual o limite pode ou não existir. 
Teorema 4.20. Sejam $f, \Gamma_{k}, \Gamma_{k n}, A_{k n}$ e $A_{k}$ definidas como em (4.14) e $N$ como acima. Se $f$ é integrável em $D$, então $N$ é um conjunto nulo.

Prova. Provaremos que $N \subset \bigcup_{k=1}^{\infty} A_{k}$.

Da integrabilidade de $f$ em $D$ sejam, para cada $n \geq 1, \delta_{n}, \mathcal{D}_{n}$, e $\phi_{n}$ satisfazendo as condições (1)-(4) de (4.19).

Mostraremos que se $z \in N$, então existe um $k \geq 1$ tal que $z \in A_{k}$.

Dado $z \in N$, existe um número natural $k$ tal que, para cada $n \geq 1$, existe um $j_{n} \geq n$, verificando

$$
\left|\phi_{j_{n}}(z)-f(z)\right| \geq \frac{1}{k}
$$

Por (4.19.(4)) existe um par $\left(x_{j(n)}, E_{s\left(j_{n}\right)}\right) \in \mathcal{D}_{n}$ tal que $z \in E_{s\left(j_{n}\right)} \mathrm{e}$

$$
\phi_{n}(z)=\frac{\int_{E_{s\left(j_{n}\right)}} f}{s\left(j_{n}\right)}
$$

Portanto, de (1) e (2)

$$
\begin{aligned}
\left|\int_{E_{s\left(j_{n}\right)}} f-s\left(j_{n}\right) \cdot f(z)\right| & =\left|\frac{\int_{E_{s\left(j_{n}\right)}} f}{s\left(j_{n}\right)}-f(z)\right| \cdot s\left(j_{n}\right) \\
& =\left|\phi_{j_{n}}(z)-f(z)\right| \cdot s\left(j_{n}\right) \\
& \geq \frac{s\left(j_{n}\right)}{k}
\end{aligned}
$$

Isso mostra, lembrando (4.14), que o par $\left(z, E_{s\left(j_{n}\right)}\right) \in \Gamma_{k}$. Mas, como $j_{n} \geq n$, usando (4.19.(1)) vem:

$$
E_{s\left(j_{n}\right)} \subset \delta_{j_{n}}\left(x\left(j_{n}\right) \subset B_{p^{-j_{n}}}\left(x\left(j_{n}\right)\right) \subset B_{p^{-n}}\left(x\left(j_{n}\right)\right)=B_{p^{-n}}(z)\right)
$$

provando que o par $\left(z, E_{s\left(j_{n}\right)}\right) \in \Gamma_{k n}$. Segue-se que $z \in A_{k n}$. mas esta condição é válida para cada $n \geq 1$. Logo $z \in \bigcup_{n=1}^{\infty} A_{k n}=A_{k}$.

Mostramos que, para cada $z \in N$, existe um $k \geq 1$ tal que $z \in A_{k}$. Seguese daí que $N \subset \bigcup_{k=1}^{\infty} A_{k}$, e, por (4.18) $N$ é um conjunto nulo, completando a prova.

Corolário 4.21. Se $f$ é integrável em $D$ então $f$ é mensurável. 
Prova. De $f$ é integrável segue-se pelo teorema acima que existe uma seqüência de funções escadas que convergem para $f$ exceto para os pontos de um conjunto nulo. Estando satisfeitas as condições de (4.1) segue-se que $f$ é mensurável.

Corolário 4.22. Toda função contínua é mensurável.

Prova. Imediata

Teorema 4.23. Se $\int_{D} \chi_{N}=0$, então $N$ é um conjunto nulo.

Prova. Dado $\varepsilon>0$ tomemos $0<\varepsilon^{\prime}<\varepsilon$ e, para esse $\varepsilon^{\prime}$, por hipótese; existe um gauge $\delta$ satisfazendo a definição da integral.

Admitamos que o raio de $D$ seja $r=p^{m}$, com $m \in \mathbb{Z}$, e seja $\alpha \in \mathbb{Z}$ tal que $p^{-\alpha}<\min \left\{p^{m}, \varepsilon^{\prime}\right\}$. Dessa forma, por (1.28), para cada $n \geq 1$ o disco $D$ é coberto por $p^{\alpha+n+m}$ discos de raio $p^{-(\alpha+n)}$.

Designemos por $\mathcal{F}_{n}$ cada uma dessas famílias de discos.

Para cada disco $E \in \mathcal{F}_{1}$ para o qual existe pelo menos um $x \in N$ satisfazendo $E \subset \delta(x)$, associemos um único desses $x$ e o designemos por $x_{E}$. Isso posto chamemos de $\mathcal{G}_{1}$ o conjunto dos pares $\left(x_{E}, E\right)$.

É imediato que $\mathcal{G}_{1}$ é finito.

Suponhamos definidos os conjuntos $\mathcal{G}_{1}, \ldots, \mathcal{G}_{n-1}$. Definamos $\mathcal{G}_{n}$. Para cada disco $G \in \mathcal{F}_{n}, n>1$, para o qual existe pelo menos um $x \in N$ satisfazendo $G \subset \delta(x)$ e tal que

$$
G \cap \bigcup_{\substack{k=1 \\\left(x_{E}, E\right) \in \mathcal{G}_{k}}}^{n-1} E=\emptyset
$$

associemos um único $x_{G} \in N$ satisfazendo essa condição e designemos

$$
\mathcal{G}_{n}=\left\{\left(x_{G}, G\right): x_{G} \in N, G \in \mathcal{F}_{n}, G \subset \delta\left(x_{G}\right), G \cap \bigcup_{\substack{k=1 \\\left(x_{E}, E\right) \in \mathcal{G}_{k}}}^{n-1} E=\emptyset\right\}
$$

Novamente $\mathcal{G}_{n}$ é finito. Segue-se que $\left\{(x, E):(x, E) \in \bigcup_{n=1}^{\infty} \mathcal{G}_{n}\right\}$ é enumerável; assim podemos indexar seus elementos obtendo a seqüência $\left(\left(x_{k}, E_{r_{k}}\right)\right)_{k \geq 1}$ com os raios $r_{k}$ satisfazendo a condição $r_{k} \geq r_{k+1}$, para todo $k \geq 1$. 
Afirmamos que

$$
N \subset \bigcup_{k=1}^{\infty} E_{r_{k}}
$$

De fato, dado $z \in N$ temos que $\delta(z)$ é um aberto, logo existe $n \in \mathbb{Z}$ satisfazendo

$$
z \in B_{p^{-(\alpha+n-1)}}(z)=D_{p^{-(\alpha+n)}}(z) \subset \delta(z) .
$$

Segue-se que apenas uma das asserções abaixo é válida

$$
D_{p^{-(\alpha+n)}}(z) \cap \bigcup_{r_{k}>p^{-(\alpha+n)}} E_{r_{k}} \neq \emptyset
$$

$\mathrm{ou}$

$$
D_{p^{-(\alpha+n)}}(z) \cap \bigcup_{r_{k}>p^{-(\alpha+n)}} E_{r_{k}}=\emptyset
$$

Se (2) ocorre, então existe um $r_{j}>p^{-(\alpha+n)}$ tal que $D_{p^{-(\alpha+n)}} \cap E_{r_{j}} \neq \emptyset$. Por (1.5.i), $D_{p^{-(\alpha+n)}} \subset E_{r_{j}}$ e, portanto $z \in \bigcup_{k=1}^{\infty} E_{r_{k}}$.

Se (3) ocorre, como $D_{p^{-(\alpha+n)}} \subset \delta(z)$, então ou $\left(z, D_{p^{-(\alpha+n)}}\right) \in \mathcal{G}_{n}$ ou existe um $y \in N$, com $y \neq z$, tal que $\left(y, D_{p^{-(a+n)}}\right) \in \mathcal{G}_{n}$. Em qualquer um desses casos temos que $D_{p^{-(\alpha+n)}}$ é igual a um disco $E_{r_{k}}$, com $r_{k}=p^{-(\alpha+n)}$. Novamente, $z \in \bigcup_{k=1}^{\infty} E_{r_{k}}$. Portanto $N \subset \bigcup_{k=1}^{\infty} E_{r_{k}}$ completando a prova de (1).

Resta verificarmos que $\sum_{k=1}^{\infty} r_{k}<\varepsilon$.

Inicialmente, lembrando (2.16.iii) temos

$$
0 \leq \int_{E_{r_{k}}} \chi_{N}=\int_{D} \chi_{N} \cdot \chi_{E_{r_{k}}} \leq \int_{D} \chi_{N}=0
$$

para todo $k \geq 1$.

Qualquer que seja $\ell \geq 1$ temos $\mathcal{D}=\left\{\left(x_{1}, E_{r_{1}}\right), \ldots,\left(x_{\ell}, E_{r_{\ell}}\right)\right\}<<<\delta$. Pelo Lema de Henstock (i), vem

$$
\left|\sum_{k=1}^{\ell}\left(\chi_{N}\left(x_{k}\right) r_{k}-\int_{E_{r_{k}}} \chi_{N}\right)\right| \leq \varepsilon^{\prime}
$$

ou

$$
\sum_{k=1}^{\ell} r_{k} \leq \varepsilon^{\prime}, \text { para todo } \ell \geq 1
$$


Portanto

$$
\sum_{k=1}^{\infty} r_{k} \leq \varepsilon^{\prime}<\varepsilon
$$

satisfazendo as condições (i) e (ii) da (3.1). Segue-se que $N$ é um conjunto nulo.

O teorema acima e (3.6) podem ser sintetizados em: $N$ é um conjunto nulo se e somente se $\int_{D} \chi_{N}=0$.

Teorema 4.24. Sejam $f$ integrável em $D$, com $f \geq 0$ q.s., e $A \in \mathcal{A}$. Então $f \chi_{A}$ é integrável em $D$.

Prova. Decorre da hipótese que $\chi_{A}$ é integrável em $D$ e, por (2.12.ii), $n \cdot \chi_{A}$ é integrável em $D$ para todo $n \geq 1$. Segue-se, por (2.31), que $f \wedge n \chi_{A}$ é integrável e a seqüência $\left(f \wedge n \chi_{A}\right)_{n \geq 1}$ satisfaz as condições: $0 \leq(f \wedge$ $\left.n \chi_{A}\right)(x) \leq f(x)$, para todo $x \in D$ e $\lim _{n \rightarrow \infty} f \wedge n \chi_{A}=f \chi_{A}$. Portanto, pelo TCD a função $f \chi_{A}$ é integrável em $D$.

Corolário 4.25. Se $f$ é absolutamente integrável em $D$ e $A \in \mathcal{A}$ então existem as integrais $\int_{A} f$ e $\int_{A}|f|$.

Prova. A integração de $|f|$ decorre do teorema anterior.

Definindo-se, para todo $x \in D, f^{+}(x)=\frac{|f|(x)+f(x)}{2}$ e $f^{-}(x)=\frac{|f|(x)-f(x)}{2}$, é imediato que $f^{+}$e $f^{-}$são não negativas. Logo, pelo teorema anterior, existem as integrais $\int_{D} f^{+} \chi_{A}$ e $\int_{D} f^{-} \chi_{A}$. O resultado segue de $f \chi_{A}=f^{+} \chi_{A}-f^{-} \chi_{A}$, usando (2.12).

A condição da função ser absolutamente integrável nos teoremas acima é essencial para a existência da integral, restrita a um conjunto mensurável, como mostra o exemplo abaixo.

Exemplo 4.26. Em $\mathbb{Z}_{3}$ seja $A$ a reunião de todos os discos de raio $3^{-2 j}$ contendo os pontos da forma $a_{0}+\ldots+a_{2 j-1} 3^{2 j-1}+2 \cdot 3^{2 j}+\ldots$, para todo $j \geq 0$ e no qual os coeficientes $a_{0}, \ldots, a_{2 j-1}$ são distintos de 2 . É imediato 
que, para todo $x \in D$, temos

$$
f \chi_{A}(x)= \begin{cases}\frac{1}{2 j+1} \cdot \frac{3^{2 j+1}}{2^{2 j}} & \text { se } x \in A \\ 0 & \text { fora }\end{cases}
$$

Definindo-se a seqüência $\left(g_{n}\right)_{n \geq 1}$ em $D$ por $g_{n}(x)=f_{n} \chi_{A}(x)$, para todo $x$, com $f_{n}$ dada em (2.11) verificamos que, para todo $x \in D$

a) $g_{n}(x) \rightarrow f \chi_{A}(x)$

b) $g_{n}(x) \leq g_{n+1}(x)$;

Pela definição das funções $f_{n}$ temos que, para cada $n \geq 1$ as funções $g_{n}$ são integráveis em $D$ e

$$
\lim _{n \rightarrow \infty} \int_{D} g_{n}=\sum_{n=1}^{\infty} \frac{1}{2 n+1}=\infty
$$

Portanto, pelo TCM, $f \chi_{A}$ não é integrável. $\diamond$ 


\section{Capítulo 5}

\section{Aplicação da Integral}

\subsection{Equação de Fredholm}

O escopo desta seção é resolver equações integrais, aplicando a teoria desenvolvida. Mostraremos que a equação integral Fredholm de segunda espécie tem solução em $D$.

Inicialmente apresentaremos alguns conceitos e notações já conhecidos da Análise Funcional. Como usualmente se procede, $C(D)$ denotará o conjunto da funções contínuas definidas no disco $D$, com valores reais. Como $D$ é um espaço completo então sabemos que

$$
\|f\|=\sup \{|f(x): x \in D|\}
$$

é um norma em $D$ e que, com essa norma, $C(D)$ é, também, um espaço completo(Veja [16]. capítulo 1). Finalmente, como de costume, definimos as funções $f_{x}, f^{y}: D \rightarrow \mathbb{R}$ por

$$
f_{x}(y)=f(x, y), \text { para todo } x \in D
$$

$\mathrm{e}$

$$
f^{y}(x)=f(x, y), \text { para todo } y \in D
$$


Lema 5.1. Sejam $f$ e $g$ integráveis e limitadas inferiormente ou superiormente em $D$. Então $f-g$ é absolutamente integrável cm D.

Prova. Admitamos que $f(x) \geq m$, para todo $x \in D$ e provemos que ela é absolutamente integrável. É claro que a função $h(x)=f(x)-m>0$ é integrável em $D$, segue-se que $h_{1}(x)=h(x)+|m|$ também é integrável em $D$. De $|f(x)|=|h(x)+m| \leq h(x)+|m|=h_{1}(x)$ vem, por (2.30) que $|f|$ é integrável em $D$. Analogamente verificamos que $g$ é absolutamente integrável.

Finalmente de $|f-g| \leq|f|+|g|$ segue-se,novamente por (2.30), que $f-g$ é absolutamente integrável.

Se $f$ for limitada superiormente escolhemos $M$ tal que $h(x)=M-f(x)>$ 0 e procedemos de forma análoga.

Teorema 5.2. (Teorema do Ponto Fixo de Banach) Sejam $X$ um espaço métrico completo e $T: X \rightarrow X$ uma contração. Então:

(i) existe um e um só $\bar{x} \in X$ tal que $T \bar{x}=\bar{x}$;

(ii) qualquer que seja $x_{1} \in X$, a seqüência $\left(x_{n}\right)_{n \in \mathbb{N}}$, onde $x_{n+1}=T^{n} x_{1}$, converge a $\bar{x}$; (iii) para todo $n$, temos $d\left(x_{n}, \bar{x}\right) \leq c^{n-1} \frac{d\left(x_{1}, x_{2}\right)}{1-c}$, onde $c$ é uma constante de contração de $T$, e $\left(x_{n}\right)_{n \in \mathbb{N}}$ é a seqüência de (ii).

Prova. Vide ([17])

Teorema 5.3. Seja $f: D \times D \rightarrow \mathbb{R}$ satisfazendo as seguintes condições:

(i) $\int_{D} f_{x}(y) d y$ existe, para cada $x \in D$;

(ii) $f_{x}$ é limitada inferiormente para cada $x \in D$;

(iii)a família $\left(f^{y}\right)_{y \in D}$ é equicontínua em $D$

(iv) $\lambda=\frac{1}{M+1}$, sendo $M=\sup \left\{\int_{D}\left|f_{x}(y)\right| d y: x \in D\right\}<\infty$.

Então, para cada $g_{0} \in C(D)$ existe uma única $g \in C(D)$ tal que

$$
g(x)=g_{0}(x)+\lambda \int_{D} f(x, y) g(y) d y
$$




\section{Prova.}

Dadas $g, g_{0} \in C(D)$ seja

$$
T g(x)=g_{0}(x)+\int_{D} f_{x}(y) g(y) d y
$$

$T g$ está bem definida pois, de (ii) e (2.25), a integral existe para cada $x \in D$.

Provemos que $T g \in C(D)$.

Dados $x_{1}, x_{2} \in D$ temos

$$
\begin{aligned}
\left|T g\left(x_{1}\right)-T g\left(x_{2}\right)\right|= & \left|g_{0}\left(x_{1}\right)-g_{0}\left(x_{2}\right)+\int_{D}\left[f_{x_{1}}(y)-f_{x_{2}}(y)\right] g(y) d y\right| \\
& \leq\left|g_{0}\left(x_{1}\right)-g_{0}\left(x_{2}\right)\right|+\left|\int_{D}\left[f_{x_{1}}(y)-f_{x_{2}}(y)\right] g(y) d y\right|
\end{aligned}
$$

Por (5.1) $f_{x_{1}}-f_{x_{2}}$ é absolutamente integrável e a continuidade de $g$ implica a continuidade de $|g|$. Logo, de (2.25) e $|f g|=|f||g|$, temos que $\left[f_{x_{1}}-f_{c_{2}}\right] \cdot g$ é absolutamente integrável. De (2) vem

$$
\left|T g\left(x_{1}\right)-T g\left(x_{2}\right)\right| \leq\left|g_{0}\left(x_{1}\right)-g_{0}\left(x_{2}\right)\right|+\left|\int_{D}\left[f^{y}\left(x_{1}\right)-f^{y}\left(x_{2}\right)\right] g(y) d y\right|
$$

Dado $\varepsilon>0$ existe $n \geq 1$ tal que $\left|x_{1}-x_{2}\right|<p^{-n}$ implica

$$
\left|g_{0}\left(x_{1}\right)-g_{0}\left(x_{2}\right)\right|<\frac{\varepsilon}{2}
$$

e, utilizando (iii),

$$
\left|f^{y}\left(x_{1}\right)-f^{y}\left(x_{2}\right)\right|<\frac{\varepsilon}{2\left[\int_{D}|g|+1\right]}
$$

De (3), (4) e (5) vem $\left|T g\left(x_{1}\right)-T g\left(x_{2}\right)\right|<\frac{\varepsilon}{2}+\frac{\varepsilon}{2\left[\int_{D}|g|+1\right]}<\varepsilon$ mostrando que $T g \in C(D)$. e o operador $T$

Provemos que $T$ é uma contração.

De fato, dados $g$ e $h$ em $C(D)$ temos, para todo $x \in D$,

$$
\begin{aligned}
\|T g(x)-T h(x)\| & =|\lambda| \cdot\left|\int_{D} f(x, y)[g(y)-h(y)] d y\right| \\
& \leq|\lambda| \int_{D}|f(x, y)| \cdot|g(y)-h(y)| d y \leq|\lambda|\|g-h\| \int_{D}\left|f_{x}(y)\right|
\end{aligned}
$$


Finalmente, usando (iv), temos que

$$
\|T g(x)-T h(x)\|<\frac{1}{M+1} \cdot \int_{D}\|g-h\| \cdot\left|f_{x}(y)\right|<\|g-h\|
$$

Isso mostra que $T$ é uma contração. Portanto $T$ tem um ponto fixo, ou seja, existe $g \in C(D)$ satisfazendo $\left({ }^{*}\right)$ e completando a prova do teorema. 


\section{Referências Bibliográficas}

[1] R.G.Bartle, Return to the Riemann Integral, American Mathematical Monthly,(1996),p.625-632

[2] R.G.Bartle, A Convergence Theorem for Generalized Riemann Integrals, Real Analysis Exchange, vol. 20(1), 1994/95, p. 119-124.

[3] R. Bianconi, On the convergence of the Integrals of a Truncated Henstock-Kurzweil Integrabel Function, Real Analysis Exchange, Vol.23(1),(1997/8),p.247-250

[4] S.Bosch, U. Güntzer \& R.Remmert, Non-Archimedean Analysis, Springer-Verlag, 1984.

[5] E. Cabral \& P.Y.Lee, A Fundamental Theorem of Calculus for the Kurzweil-Henstock integral in $\mathbb{R}^{m}$, Real Analysis Exchange, vol.26(2),(2000/01),p.867-876

[6] E. Cabral \& P.Y.Lee, The Primitive of a Kurzweil-Henstock integrable function in multidimensional space, Real Analysis Exchange, vol.27(2),(2001/02),p.627-634

[7] D.J.Cohn,Measure Theory, Birkhäuser,Boston, 1980

[8] J.D.DePree and C.W.Swartz, Introduction to the real analysis, John Wiley \& Sons Inc., New York, 1988.

[9] E.L.Lima, Espaços Métricos, IMPA, Rio de Janeiro, 1977

[10] M. Federson, Two equivalent definitions of the Kuтzweil integral, $49^{\circ}$ Seminário Brasileiro de Análise, (1999), 723-730. 
[11] I.Fleischer, A Vitali-like Convergence Theorem for the Henstock Integral, Real Analysis Exchange,Vol.32(1996/7),p.174-176

[12] R.A Gordon, The Integrals of Lebesgue, Denjoy, Perron, and Henstock, Graduate Studies in Mathematics, $N^{\circ} 4$, American Mathematical Society, 1994.

[13] R.A Gordon, A Convergence Theorem for the Riemann Integral,Mathematics Magazine, v. 73, $N^{\circ} 2$, April 2000.

[14] F.Q.Gouvêa, p-adic Numbers, Springer-Verlag, 1991.

[15] C.S. Hönig, As integrais de Gauge, $37^{\circ}$ Seminário Brasileiro de Análise (1993), 3-59.

[16] C.S. Hönig, Análise Funcional e Aplicações- v.1 e 2, Publicações do IME-USP,São Paulo (1970)

[17] C.S. Hönig, Aplicações da topologia à análiseIMPA,CNPQ, Rio de Janeiro(1976)

[18] L. Jitan \& L.P.Yee, The primitives of Henstock Integrable Function in Euclidean Space, Bull. London Math.Soc.31 (1999), p. 173-180.

[19] N.Koblitz, p-adic Numbers, p-adic Analysis and Zeta-Functions, Springer-Verlag.

[20] J. Kurzweil, A nonexistence result for the Kurzweil integral, Math. Bohemia 127(2002) No4, 571-580.

[21] L.P. Yee \& R.V ýborný, The Integral: An Easy Approach after Kurzweil and Henstock, Austr.Math. Soc. Lecture Series, v.14, Lnited Kingdom, 2000 .

[22] P.Y.Lee, Lanzhou Lectures on Henstock Integration, World Scientific, Singapure, 1989

[23] P.Y.Lee, Z.M. Ma,T.S.Chew,Absolute Integration Using Vitali Covers, Real Analysis Exchange,Vol.18(2)(1992/93),p.409-419

[24] E.L.Lima, Espaços Métricos,Edgard Blücher Ltda.,São Paulo, 1977. 
[25] G. Liu, On Necessary Conditions for Henstock Integrability, Real Analysis Exchange, Vol. 18(2), (1992/93), 522-531.

[26] J.Mawhin, Introduction a l'Analyse,Cabay, 1984.

[27] R.M.McLeod, The Generalized Riemann Integral, Carus Mathematical Monographs, $N^{\circ}$ 20, Mathematical Association of America, 1980.

[28] E.J.McShane, A Unified Theory of Integration, American Mathematical Monthly, 80 (1973), 349-359.

[29] A.P. Monna,Analyse non-archimédienne, Springer-Verlag, 1970.

[30] S.Schwabik, Generalized Ordinary Differential Equations, World Scientific,Singapure, 1992.

[31] S.Schwabik,Henstock's Condition for Convergence Theorems and Equiintegrability, Real Analysis Exchange, Vol.18(1)(1992/93), 190-205

[32] L.Shipan, L.Kecheng, On Generalized Dominated Convergence,Real Analysis Exchange, vol.16 (1990/91)

[33] L.Shi-pan, L.Peng-Yee, Globally Small Riemann Sums and the Henstock Integral,Real Analysis Exchange, vol.16 (1990/91)

[34] C.Swartz, Introduction to Gauge Integrals, World Scientific,Singapure, 2001

[35] L. Tuo-Yeong, A Full Descriptive Definition of the Henstock-Kurzweil Integral in the Euclidean Space, Proc. London Math. Soc., 87(3), 2003, $677-700$

[36] V.S.Vladimirov,I.V. Volovich \& E.I.Zelenov, p-adic Analysis and Mathematical Physics, World Scientific, Singapure, 1994. 